\title{
A Fuzzy Expectation Maximization based method for estimating the parameters of a multi-state degradation model from...
}

Article in Annals of Nuclear Energy · July 2017

DOI: 10.1016/j.anucene.2017.07.017

CITATIONS

0

4 authors, including:

Francesco Cannarile

Politecnico di Milano

6 PUBLICATIONS 6 CITATIONS

SEE PROFILE

\section{Enrico Zio}

Politecnico di Milano

827 PUBLICATIONS 9,184 CITATIONS

SEE PROFILE
READS

60

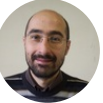

\section{Michele Compare}

Politecnico di Milano

48 PUBLICATIONS 241 CITATIONS

SEE PROFILE

Some of the authors of this publication are also working on these related projects: 


\title{
A Fuzzy Expectation Maximization based method for estimating the parameters of a multi-state degradation model from imprecise maintenance outcomes
}

\author{
F. Cannarile ${ }^{1,2}$, M. Compare $^{1,2}$, E. Rossi ${ }^{1}$, E. Zio ${ }^{1,2,3}$ \\ ${ }^{1}$ Dipartimento di Energia, Politecnico di Milano, Italy \\ ${ }^{2}$ Aramis s.r.l., Milano, Italy
}

${ }^{3}$ Chair on Systems Science and Energetic Challenge, Foundation Electricité de France at Ecole CentraleSupelec, France

\begin{abstract}
Multi-State (MS) reliability models are used in practice to describe the evolution of degradation in industrial components and systems. To estimate the MS model parameters, we propose a method based on the Fuzzy Expectation-Maximization (FEM) algorithm, which integrates the evidence of the field inspection outcomes with information taken from the maintenance operators about the transition times from one state to another. Possibility distributions are used to describe the imprecision in the expert statements. A procedure for estimating the Remaining Useful Life (RUL) based on the MS model and conditional on such imprecise evidence is, then, developed. The proposed method is applied to a case study concerning the degradation of pipe welds in the coolant system of a Nuclear Power Plant (NPP). The obtained results show that the combination of field data with expert knowledge can allow reducing the uncertainty in degradation estimation and RUL prediction.
\end{abstract}

Key words: Multi-State Systems, Homogeneous Continuous-Time Semi-Markov Process (HCTSMP), Weibull Distribution, Fuzzy Expectation-Maximization (FEM), Residual Useful Life (RUL), Piping System (PS), Nuclear Power Plants (NPPs).

\section{Acronyms and symbols}

CDF Cumulative Distribution Function

EM Expectation-Maximization

FEM Fuzzy Expectation-Maximization

HCTSMM Homogeneous Continuous-Time Semi-Markov Model

MC Monte Carlo 
MS

Multi-State

MLE Maximum Likelihood Estimation

NPP Nuclear Power Plant

PDF Probability Density Function

PFM Probabilistic Fracture Mechanics

PS Piping System

PWR Pressurized Water Reactor

RCS Reactor Cooling System

RUL Remaining Useful Life

$C_{0} \quad$ Case 0

$C_{1} \quad$ Case 1: moderately risk-averse expert

$C_{2} \quad$ Case 2: risk averse expert

$C_{3} \quad$ Case 3: risk prone expert

$D \quad$ Dataset of inspection outcomes

E State space

$f_{T_{i \rightarrow i+1}} \quad$ PDF of $T_{i \rightarrow i+1}$

$f_{T_{i \rightarrow i+1}}\left(\cdot \mid t_{n, i}^{0}\right)$ Conditional PDF of $T_{i \rightarrow i+1}$ provided that $T_{i \rightarrow i+1} \geq t_{n, i}^{0}$

$f_{R U L(k \tau)} \quad$ PDF of $R U L(k \tau)$

$F_{T_{i \rightarrow i+1}} \quad$ CDF of $T_{i \rightarrow i+1}$

$F_{\text {failure }} \quad \mathrm{CDF}$ of $T^{\text {failure }}$

$\tilde{f}_{T_{i \rightarrow i+1}} \quad$ PDF of fuzzy observations

$k_{n, i} \quad$ Inspection at which the $n^{\text {th }}$ component is found in state $i$ for the first time

$L \quad$ Likelihood function

$\tilde{L} \quad$ Likelihood function of fuzzy observations

$\mathcal{L}_{i \rightarrow i+1} \quad i^{\text {th }}$ contribution to the log-likelihood function

$\tilde{\mathcal{L}}_{i \rightarrow i+1} \quad i^{\text {th }}$ contribution to log-likelihood function of fuzzy observations

$\mathcal{L}_{\log } \quad$ Log-likelihood function

$\tilde{\mathcal{L}}_{\log } \quad$ Log-likelihood function of fuzzy observations

$M_{n} \quad$ Number of inspections on component $n$

$N \quad$ Number of components

$Q \quad$ Log-likelihood function conditional on fuzzy evidence

$R_{T_{i \rightarrow i+1}} \quad$ Reliability function of $T_{i \rightarrow i+1}$

$R^{\text {failure }} \quad$ Reliability function of $T^{\text {failure }}$

$t \quad$ Time

$\boldsymbol{t} \quad$ Transition time dataset 
$t_{n} \quad$ Vector of transition times of the $n^{\text {th }}$ component

$T_{i \rightarrow i+1} \quad$ Transition time from state $i$ to state $i+1$, random variable

$T_{m} \quad$ Mission time

$T^{\text {failure }} \quad$ Failure time

$t_{n, i \rightarrow i+1} \quad$ Transition time of the $n^{\text {th }}$ component from state $i$ to state $i+1$, observed value

$\tilde{t}_{n, i \rightarrow i+1} \quad$ Fuzzy transition time, observed value

$\underline{t}_{n, i \rightarrow i+1} \quad$ Lower bound of the support of $\mu_{\tilde{t}_{n, i \rightarrow i+1}}\left(t_{i \rightarrow i+1}\right)$

$\stackrel{m}{t}_{n, i \rightarrow i+1} \quad$ Core of $\mu_{\tilde{t}_{n, i \rightarrow i+1}}\left(t_{i \rightarrow i+1}\right)$

$\bar{t}_{n, i \rightarrow i+1} \quad$ Upper bound of the support of $\mu_{\tilde{t}_{n, i \rightarrow i+1}}\left(t_{i \rightarrow i+1}\right)$

$t_{n, i}^{0}$

$\dot{t}_{n, i}^{0}$

Sojourn time in state $i$ of the $n^{\text {th }}$ component

Elapsed time from the first inspection time in which the component has been found in state $i$, and the last one

$\alpha_{i} \quad$ Scale parameter of the Weibull distribution describing the uncertainty on the transition time from state $i$ to state $i+1$

$\beta_{i} \quad$ Shape parameter of the Weibull distribution describing the uncertainty on the transition time from state $i$ to state $i+1$

$\boldsymbol{\delta} \quad$ Vector of $\delta^{n}, n=1 \ldots N$

$\delta_{n} \quad$ Vector of binary variables associated to the $n^{\text {th }}$ component

$\delta_{n, i \rightarrow i+1} \quad$ Binary variable associated to the $n^{\text {th }}$ component indicating the censoring of the transition time from state $i$ to state $i+1$

$\lambda_{i \rightarrow i+1} \quad$ Transition rate from state $i$ to state $i+1$

$\lambda_{C 0} \quad$ Transition rate for Case $C 0$

$\lambda_{C 2} \quad$ Transition rate for Case $C 2$

$\lambda_{C 3} \quad$ Transition rate for Case $C 3$

$\mu_{\tilde{t}_{n, i \rightarrow i+1}} \quad$ Possibility distribution on $\tilde{t}_{n, i \rightarrow i+1}$

$\tau \quad$ Interval between two successive inspections

$\vartheta \quad$ Vector of the transition time parameters vector

$\hat{\vartheta}_{\text {mle }} \quad$ MLE estimates of $\vartheta$

$\vartheta^{q} \quad$ Estimates of $\vartheta$ at iteration $q$

$i \quad$ state index, $i=1,2,3$

$k \quad$ inspection index, $k=1 \ldots M_{n}$

$n \quad$ component index, $n=1 . . N$

$q \quad$ FEM iteration index

\section{INTRODUCTION}

Multi-State (MS) degradation modelling is receiving considerable attention in the domain of reliability and maintenance engineering (Zio, 2016), due the fact that MS models offer a description 
of the degradation evolution which is more realistic than that given by binary models: the evolution of many degradation processes proceeds in successive phases, which reflect the relative degree of deterioration (Moghaddass \& Zuo, 2014). A further reason which justifies the growing interest in MS degradation models is their fit with the field maintenance data acquired from the operating systems. For example, operators typically assign a qualitative tag to the equipment health during periodic inspections such as 'not degraded', 'slightly degraded', 'badly degraded', etc.

Given these characteristics, MS models have been adopted to describe the evolution of degradation of components of diverse application fields: membranes of pumps operating in Nuclear Power Plants (NPPs) (Baraldi et al., 2011), turbine nozzles for the Oil\&Gas industry (Compare et al., 2016), turbofan engines (Moghaddas \& Zuo, 2014), Diesel engines (Giorgio et al., 2011), to cite a few.

A Multi-State (MS) degradation model has also been developed in (Fleming and Smit, 2008) for the Piping System (PS) of NPPs, where PSs are highly risk-sensitive structural elements (Gopika et al., 2003; Di Maio et al., 2015). In details, in the model by (Fleming \& Smit, 2008), which is general enough to represent all known NPP pipe failure mechanisms (Fleming, 2004), the degradation process affecting a PS is discretized into four states, each one associated to a physically different phenomenon, with state transition rates that are taken constant over time and, consequently, sojourn times in each state that obey exponential distributions (e.g., Fleming \& Smit, 2008). However, it has been shown in (Veeramany \& Pandey, 2011; Chatterjee \& Modarres, 2008), that the constant rate assumption is not coherent with the evidence coming from many real industrial applications. Thus, to overcome the limitation of constant transition rates, the theoretical framework of the Homogeneous Continuous-Time Semi-Markov Processes (HCTSMPs, Howard, 1964) has been embraced to develop MS degradation models, which allow considering arbitrary sojourn time distributions, thus, taking into account the influence of the history of the degradation process on its future evolution. In particular, (Veeramany \& Pandey, 2011) developed a HCTSMP model to describe the degradation of PSs in NPPs.

For practical application, the estimation of the parameters of the MS semi-Markov degradation model, with associated uncertainty, is fundamental and different approaches have been proposed in the literature to adjust the model to the knowledge, information and data available.

When sufficient field data is available, statistical techniques such as Maximum Likelihood Estimation (MLE) can be adopted (Zio, 2007; Gosselin \& Fleming, 1997). However, the availability of rich datasets of NPP PS degradation and maintenance data is not typical and the problem of parameter estimation is further complicated by at least two other aspects: 
- The inherent complexity of the PSs in NPPs and diversity in the degradation influenced by operating and ambient conditions (Tipping, 2010); then, it becomes difficult to identify mechanisms and homogeneous populations of PS for statistical inference.

- The possible noninformativeness of the data, i.e., of the outcomes of inspections performed every 2-5 years, in which the PS is typically found in the first degradation states, due to its very high reliability (Nánási, T., 2014; Fleming, 2004; Veeramany \& Pandey, 2011; Simonen \& Goselin, 2001).

With this scarcity of data, it is necessary to exploit any additional knowledge or information available to build more accurate reliability models (Zio, 2016). In this respect, Probabilistic Fracture Mechanics (PFM) models have been developed to predict PS crack initiation and growth from existing flaws (Verma \& Srividya, 2011), which combine the knowledge about the physics of the crack propagation, modelled as a stochastic process, with PS service data that are used to tune the PFM model parameters. However, (Fleming, 2004) pointed out that one main limitation of the PFM approach is that the data used for model setting reflect the influence of previous PS inspection programs; thus, changes in these programs may introduce biases in the transition rates estimates.

In the present work, we consider that additional information on the occurrence of state transitions can be obtained from experts to supplement field data. Namely, we assume that experts can give statements such as "The pipe transition from detectable flaw state to detectable leak state occurred between 1998 and 2000, March 1999 being the expected month for this transition". Obviously, the imprecision in these qualitative statements need to be properly represented and combined with the field data.

Bayesian statistics is often adopted to this aim, starting from the elicitation from experts of prior distributions of the model parameters and following with their update based on to the field evidence collected (Compare et al., 2017a). Markov Chain Monte Carlo algorithms (Robert \& Casella, 2004) can be used to estimate the posterior distributions of the multi-state model parameters, which encode both the prior expert knowledge and the field evidence. However, the representation by probability distributions of the imprecision in the qualitative expert statements is debatable, as it has been argued that the probabilistic approach in situations of scarce evidence tends to force assumptions that may not be justified by the available information (Aven et al., 2014; Baudrit et al., 2008; Bowles \& Peláez, 1995). For this reason, we here use possibility distributions to represent the imprecision in the expert statements about the transition times, to "imperfectly specify a value that is existing and precise, but not measurable with exactitude under the given observation conditions" (Denoeux, 2011). 
To estimate the MS model parameters from partially observed data, we resort to the Fuzzy Expectation-Maximization (FEM) algorithm (Denoeux, 2011); this uses the Zadeh's extension principle to extend the application of standard statistical approaches to possibility distributions, which are formally coincident with the membership functions of fuzzy sets (Dubois, 2006).

Finally, based on the MS degradation model, we propose a methodology to estimate the Remaining Useful Life (RUL) of the NPP PSs.

To sum up, the main contributions of the present work are:

1. The development of a methodology to estimate the parameters of a MS degradation model, which exploits both data from inspection outcomes and expert information.

2. The development of a methodology to estimate the RUL, conditional on imprecise evidence. The remainder of the paper is organized as follows. Section 2 describes the problem settings, the available information and data. Section 3 illustrates the methodology to estimate the unknown parameters of the HCTSMP model in the considered settings. In Section 4, the methodology to estimate the RUL is illustrated. The application of the developed methodologies to a case study concerning simulated PS degradation paths is reported in Section 5. Finally, Section 6 concludes the work.

\section{Problem Settings}

To focus concretely the illustration of our work, we develop a MS model derived from the 4-states model proposed by (Fleming, 2004) for describing degradation in PSs of NPPs (Figure 1).

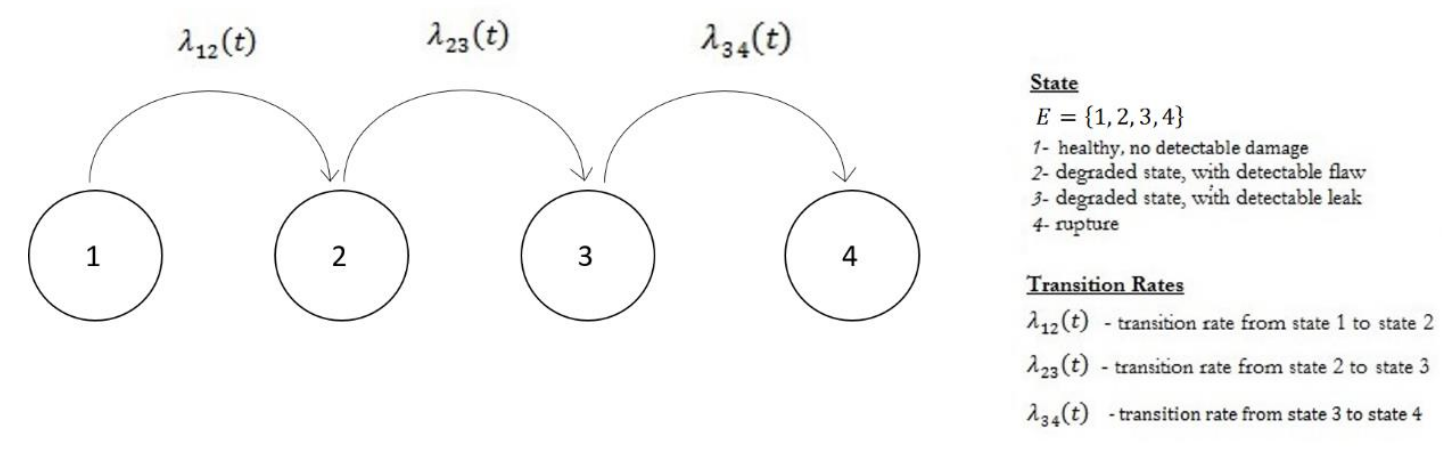

Figure 1: Sketch of MS model.

In state 1, the PS is assumed to be in an as good as new state; flaws are present but not detectable. These gradually grow until they become detectable, whose condition is represented by state 2 . Then, the PS further degrades and a leak becomes detectable (state 3). Finally, the leak extends until it leads to rupture (state 4) (Veeramany \& Pandey, 2011). Pipes are assumed to be non-repairable: this means 
that in the representation of the model (Figure 1), the transitions only go from left-to-right and also that state 4 is an absorbing state (i.e., once reached, it cannot be left).

The random transition time, $T_{i \rightarrow i+1}$, from state $i$ to state $i+1$ is assumed to obey a Weibull distribution (Cannarile et al., 2015a), with scale parameter $\alpha_{i}$ and shape parameter $\beta_{i}, i=1,2,3$. The probability density function (PDF) of $T_{i \rightarrow i+1}$ is given by

$$
f_{T_{i \rightarrow i+1}}\left(t_{i \rightarrow i+1}\right)=\frac{\beta_{i}}{\alpha_{i}} \cdot\left(\frac{t_{i \rightarrow i+1}}{\alpha_{i}}\right)^{\left(\beta_{i}-1\right)} \exp \left(-\left(\frac{t_{i \rightarrow i+1}}{\alpha_{i}}\right)^{\beta_{i}}\right) \quad t_{i \rightarrow i+1}>0, \alpha_{i}>0, \beta_{i}>0
$$

and the corresponding Cumulative Distribution Function (CDF) $F_{T_{i \rightarrow i+1}}\left(t_{i \rightarrow i+1}\right)$, reliability function $R_{T_{i \rightarrow i+1}}\left(t_{i \rightarrow i+1}\right)$ and transition rate $\lambda_{T_{i \rightarrow i+1}}\left(t_{i \rightarrow i+1}\right)$ are, respectively:

$$
\begin{aligned}
& F_{T_{i \rightarrow i+1}}\left(t_{i \rightarrow i+1}\right)=\int_{0}^{t_{i \rightarrow i+1}} f_{T_{i \rightarrow i+1}}(t) d t=1-e^{-\left(\frac{t_{i \rightarrow i+1}}{\alpha}\right)^{\beta_{i}}} \\
& R_{T_{i \rightarrow i+1}}\left(t_{i \rightarrow i+1}\right)=\exp \left(-\left(\frac{t_{i \rightarrow i+1}}{\alpha_{i}}\right)^{\beta_{i}}\right) \quad t_{i \rightarrow i+1}>0, \alpha_{i}>0, \beta_{i}>0 \\
& \lambda_{T_{i \rightarrow i+1}}\left(t_{i \rightarrow i+1}\right)=\frac{\beta_{i}}{\alpha_{i}} \cdot\left(\frac{t_{i \rightarrow i+1}}{\alpha_{i}}\right)^{\left(\beta_{i}-1\right)} \quad t_{i \rightarrow i+1}>0, \alpha_{i}>0, \beta_{i}>0
\end{aligned}
$$

The choice of relying on Weibull distributions is justified by practical reasons: Weibull distributions are the probability distributions most commonly used in reliability engineering to describe the degradation processes of industrial components in the semi-Markov framework (Boutros et al., 2011) (Moghadass \& Zuo, 2012), (Compare et al. 2017b), (Giorgio et al., 2011) due to their flexibility and the clear meaning of the distribution parameters. For this reason, experts of different industrial fields feel comfortable with using Weibull distributions to characterize the evolution of the degradation processes (Cannarile et al., 2017).

\subsection{Available data}

We assume that a dataset $D$ is available containing the inspection outcomes of $N$ NPP PSs, whose degradation evolves according to the HCTSMM described above. We also assume that each 
component is perfectly working (i.e., it is in state 1 ) at time $t=0$ and is inspected with period $\tau$ over the mission time $T_{m}$.

We indicate by $M_{n}$ the number of inspections performed on the $n^{\text {th }}$ component through its mission time $T_{m}$, whereas $k_{n, i}$ represents the first inspection at which the $n^{\text {th }}$ component is found in state $i$, with $k_{n, 1}=0$ and $k_{n, i} \in\left\{1, \ldots, M_{n}\right\}, i=2,3,4$.

In this setting, the transition time $t_{n, i \rightarrow i+1}$ of the $n^{\text {th }}$ PS, $n=1 \ldots N$, from state $i$ to state $i+1, i=$ $1,2,3$, can be regarded as a realization of the random variable $T_{i \rightarrow i+1}$, induced by randomly sampling from a population of NPP PSs (Denoeux, 2011), although a censoring mechanism avoids observing transition times that would occur after the time horizon $T_{m}$. In this respect, if $i_{n}^{*}=$ $\max _{i \in\{1,2,3,4\}}\left(\sum_{j=1}^{i} t_{n, j-1 \rightarrow j}<T_{m}\right)<4$, where $t_{n, 0 \rightarrow 1}=0$, then for simplicity we set $k_{n, s+1}=M_{n}, \forall s \geq$ $i^{*} \cap s \in\{2,3,4\}$. For example, if the $n^{\text {th }}$ component is found in state 3 at the end of the mission time $T_{m}$, then $i^{*}=3, k_{n, 3}=k_{n, 4}=M_{n}$, whereas $t_{n, 3 \rightarrow 4}$ is unknown, but larger than $T_{m}-\left(t_{n, 1 \rightarrow 2}+\right.$ $\left.t_{n, 2 \rightarrow 3}\right)$.

On this basis, we introduce the binary variable for $i=1,2,3$

$$
\delta_{n, i \rightarrow i+1}=\left\{\begin{array}{c}
0 \quad \text { if } \sum_{j=1}^{i} t_{n, j-1 \rightarrow j}<T_{m} \text { and } t_{n, i \rightarrow i+1} \leq\left[T_{m}-\sum_{j=1}^{i} t_{n, j-1 \rightarrow j}\right] \\
1 \text { otherwise }
\end{array}\right.
$$

to indicate whether $t_{n, i \rightarrow i+1}$ is an actual transition time or a right-censored observation (Zio, 2007). In words, $\delta_{n, i \rightarrow i+1}$ is set to 0 if the transition from state $i$ to state $i+1$ occurred before $T_{m}$, and to 1 otherwise (Cannarile et al., 2015b).

Moreover, even for uncensored transitions, $t_{n, i \rightarrow i+1}$ cannot be directly observed; rather, we know that $\left(k_{n, i+1}-1\right) \tau \leq\left[\sum_{j=1}^{i} t_{n, j-1 \rightarrow j}+t_{n, i \rightarrow i+1}\right] \leq k_{n, i+1} \tau, k=1, \ldots, M_{n}$ and $i=1,2,3$.

Formally, the available dataset can be represented by $D=(\boldsymbol{t}, \boldsymbol{\delta})$, where $\boldsymbol{t}=\left[t_{1}, \ldots t_{N}\right], t_{n}=$ $\left[t_{n, 0 \rightarrow 1}, \ldots, t_{n, i_{n}^{*}-1 \rightarrow i_{n}^{*}}\right], \boldsymbol{\delta}=\left[\delta_{1}, \ldots, \delta_{N}\right]$, and $\delta_{n}=\left[\delta_{n, 1 \rightarrow 2}, \delta_{n, 2 \rightarrow 3}, \delta_{n, 3 \rightarrow 4}\right], n=1, \ldots, N$.

\subsection{Information from experts}

When the expert inspects the component, he/she can add additional information on the transition times, based on his/her knowledge. We assume that expert statements about the unknown $t_{n, i \rightarrow i+1}$ give: 
1. An interval $\left[\underline{t}_{n, i \rightarrow i+1}, \bar{t}_{n, i \rightarrow i+1}\right]$ in which the transition certainly occurred, where $\left[\sum_{j=1}^{i} t_{n, j-1 \rightarrow j}+\underline{t}_{n, i \rightarrow i+1}\right] \geq\left(k_{n, i+1}-1\right) \tau ;\left[\sum_{j=1}^{i} t_{n, j-1 \rightarrow j}+\bar{t}_{n, i \rightarrow i+1}\right] \leq k_{n, i+1} \tau$.

2. A time instant $\tilde{t}_{n, i \rightarrow i+1} \in\left[\underline{t}_{n, i \rightarrow i+1}, \bar{t}_{n, i \rightarrow i+1}\right]$ in which the transition occurrence is fully plausible.

For simplicity, these pieces of information are represented by triangular possibility distributions:

$$
\mathcal{L} \mu_{\tilde{t}_{n, i \rightarrow i+1}}\left(t_{i \rightarrow i+1}\right)=\left(\underline{t}_{n, i \rightarrow i+1}, \dddot{t}_{n, i \rightarrow i+1}, \bar{t}_{n, i \rightarrow i+1}\right)
$$

with support $\left[\underline{t}_{n, i \rightarrow i+1}, \bar{t}_{n, i \rightarrow i+1}\right]$ and core $\tilde{t}_{n, i \rightarrow i+1}$, (see Figure 2$)$. Namely, $\mu_{\tilde{t}_{n, i \rightarrow i+1}}\left(t_{i \rightarrow i+1}\right)$ expresses the degree of possibility that the true value of $T_{i \rightarrow i+1}$ is $t_{i \rightarrow i+1}$ : when $\mu_{\tilde{t}_{n, i \rightarrow i+1}}\left(t_{i \rightarrow i+1}\right)=0$, then the outcome $t_{i \rightarrow i+1}$ is considered impossible by the expert, whereas $\mu_{\tilde{t}_{n, i \rightarrow i+1}}\left(t_{i \rightarrow i+1}\right)=1$ means that the outcome $t_{i \rightarrow i+1}$ is fully plausible, expected by the expert (Aven et al., 2014).

Notice that the triangular shape for the possibility distribution is the appropriate choice when the expert is willing to specify the most likely value that $t_{n, i \rightarrow i+1}$ can assume (Aven et al., 2014).

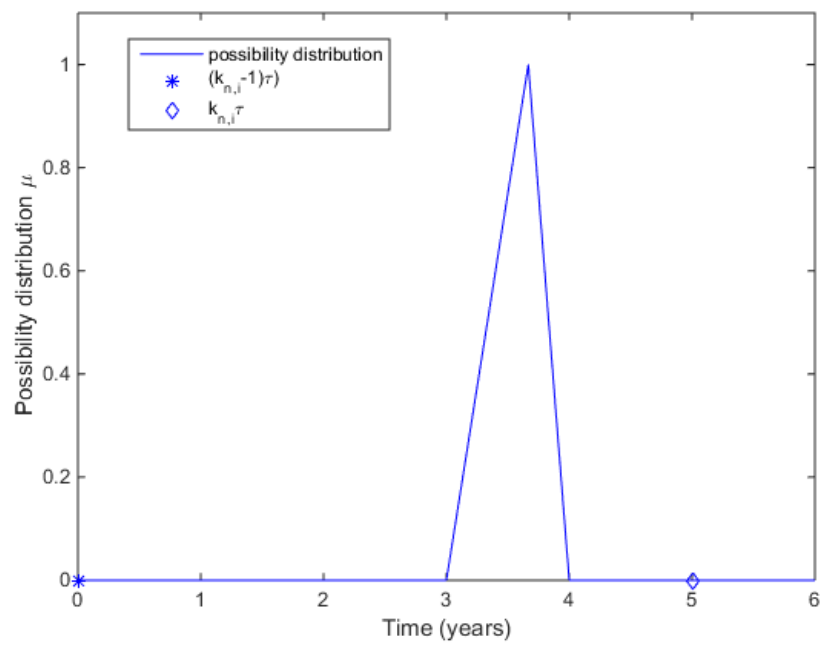

Figure 2: Possibility distribution example for the information $\underline{t}_{n, i \rightarrow i+1}=3, \tilde{t}_{n, i \rightarrow i+1}=3.667, \bar{t}_{n, i \rightarrow i+1}=4$.

\section{Parameter Estimation}

The aim of this Section is estimating the parameters $\left(\alpha_{i}, \beta_{i}\right)$ of the Weibull distributions. We consider two different situations:

- The only source of information available is dataset $D$. In this standard case, we can apply the MLE approach. 
- Also information provided by experts about transition times is available, described in the form of possibility distributions $\mu_{\tilde{t}_{n, i \rightarrow i+1}}\left(t_{i \rightarrow i+1}\right), i=1,2,3, n=1, \ldots, N$. In this case, we use the FEM algorithm proposed in (Denoeux, 2011).

The comparison of these two settings allows highlighting the benefit of exploiting the information coming from experts.

For clarity of presentation, the distribution parameters are indicated by $\vartheta=\left[\vartheta_{1}, \vartheta_{2}, \vartheta_{3}\right]$, where $\vartheta_{i}=$ $\left[\alpha_{i}, \beta_{i}\right], i=1,2,3$.

\subsection{Estimation based on inspection outcomes, only}

The application of MLE requires defining the likelihood function, which is given by:

$$
L(\vartheta \mid D)=\prod_{n=1}^{N}\left[\prod_{i=1}^{3}\left[F_{T_{i \rightarrow i+1}}\left(k_{n, i+1} \tau\right)-F_{T_{i \rightarrow i+1}}\left(\left(k_{n, i+1}-1\right) \tau\right)\right]^{\delta_{n, i \rightarrow i+1}} \cdot R_{T_{i \rightarrow i+1}}\left(T_{m}-k_{n, i} \tau\right)^{\left(1-\delta_{n, i \rightarrow i+1}\right)}\right]
$$

where the difference $F_{T_{i \rightarrow i+1}}\left(k_{n, i+1} \tau\right)-F_{T_{i \rightarrow i+1}}\left(\left(k_{n, i+1}-1\right) \tau\right)$ represents the probability of finding the component for the first time in state $i+1$ at inspection $k_{n, i+1}$, provided that it was in state $i$ at inspection $\left(k_{n, i}-1\right)$, whereas $R_{T_{i \rightarrow i+1}}\left(T_{m}-k_{n, i} \tau\right)$ indicates the probability of spending time $\left(T_{m}-k_{n, i} \tau\right)$ in state $i$. The quantity $\delta_{n, i \rightarrow i+1}$ determines which of the two contributions has to be considered for the $n^{\text {th }}$ component, depending on the censoring mechanism it has undergone.

The corresponding log-likelihood can be written as:

$$
\mathcal{L}(\vartheta \mid D)=\mathcal{L}_{1 \rightarrow 2}\left(\theta_{1} \mid D\right)+\mathcal{L}_{2 \rightarrow 3}\left(\theta_{2} \mid D\right)+\mathcal{L}_{3 \rightarrow 4}\left(\theta_{3} \mid D\right)=\sum_{i=1}^{3} \mathcal{L}_{i \rightarrow i+1}\left(\theta_{i} \mid D\right)
$$

where

$$
\begin{gathered}
\mathcal{L}_{i \rightarrow i+1}\left(\vartheta_{i} \mid D\right)=\sum_{n=1}^{N} \log \left(\left[F_{T_{i \rightarrow i+1}}\left(k_{n, i+1} \tau\right)-F_{T_{i \rightarrow i+1}}\left(\left(k_{n, i+1}-1\right) \tau\right)\right]^{\delta_{n, i \rightarrow i+1}} R_{T_{i \rightarrow i+1}}\left(T_{m}-k_{n, i} \tau\right)^{1-\delta_{n, i \rightarrow i+1}}\right) \\
\sum_{j=1}^{i} t_{n, j-1 \rightarrow j}<\left(k_{n, i}-1\right) \tau, i=1,2,3
\end{gathered}
$$

The estimation $\hat{\vartheta}_{m l e}$ of $\vartheta$ is given by: 


$$
\hat{\vartheta}_{m l e}=\underset{\vartheta \in \Theta}{\operatorname{argmax}} \mathcal{L}(\vartheta \mid D)
$$

Notice that Equations (8) - (9) simplify the estimation of $\hat{\vartheta}_{m l e}$. In fact, each of the three contributions $\mathcal{L}_{i \rightarrow i+1}, i=1,2,3$ depends on the two parameters $\left(\alpha_{i}, \beta_{i}\right)$, only. Then, one can divide the maximization problem in Equation (7) into three simpler sub-problems, which can be solved independently on each other.

\subsection{Estimation based on inspection outcomes and information elicited from experts}

To show the methodology to estimate $\vartheta$ based on the possibility distributions $\mu_{\tilde{t}_{n, i \rightarrow i+1}}$, we first derive the likelihood function as if we exactly knew the transition times $t_{n, i \rightarrow i+1}$. On this basis, we will easily extend this function to the case of imprecise transition times $\tilde{t}_{n, i \rightarrow i+1}$.

Analogously to the previous case, the likelihood function reads:

$$
L(\vartheta \mid D)=\prod_{n=1}^{N}\left[\prod_{i=1}^{3} f_{T_{i \rightarrow i+1}}\left(t_{n, i \rightarrow i+1}\right)^{\delta_{n, i \rightarrow i+1}} \cdot R_{T_{i \rightarrow i+1}}\left(T_{m}-\sum_{j=1}^{i} t_{n, j-1 \rightarrow j}\right)^{\left(1-\delta_{n, i \rightarrow i+1}\right)}\right]
$$

where the pdf $f_{T_{i \rightarrow i+1}}\left(t_{n, i \rightarrow i+1}\right)$ is used instead of $F_{T_{i \rightarrow i+1}}\left(k_{n, i+1} \tau\right)-F_{T_{i \rightarrow i+1}}\left(\left(k_{n, i+1}-1\right) \tau\right)$, because in this case we are assuming to know the transition times. Notice that the conditioning on $D$ in Equation (11) also concerns the fact that $\left(\sum_{j=1}^{i} t_{n, j-1 \rightarrow j}+t_{n, i \rightarrow i+1}\right) \in\left[\left(\sum_{j=1}^{i} t_{n, j-1 \rightarrow j}+\right.\right.$ $\left.\left.\underline{t}_{n, i \rightarrow i+1}\right),\left(\sum_{j=1}^{i} t_{n, j-1 \rightarrow j}+\bar{t}_{n, i \rightarrow i+1}\right)\right] \subseteq\left[\left(k_{n, i+1}-1\right) \tau, k_{n, i+1} \tau\right]$, if $\delta_{n, i \rightarrow i+1}=0$.

Analogously to Equation (7), Equation (11) can be divided in three parts to divide the maximization problem into three easier sub-problems and, thus, simplify the parameter estimation problem:

$$
\mathcal{L}(\vartheta \mid D)=\mathcal{L}_{1 \rightarrow 2}\left(\alpha_{1}, \beta_{1} \mid D\right)+\mathcal{L}_{2 \rightarrow 3}\left(\alpha_{2}, \beta_{2} \mid D\right)+\mathcal{L}_{3 \rightarrow 4}\left(\alpha_{3}, \beta_{3} \mid D\right)
$$

where

$$
\mathcal{L}_{i \rightarrow i+1}\left(\vartheta_{i} \mid D\right)=\sum_{n=1}^{N} \log \left[f_{T_{i \rightarrow i+1}}\left(t_{n, i \rightarrow i+1}\right)^{\delta_{n, i \rightarrow i+1}} \cdot R_{T_{i \rightarrow i+1}}\left(T_{m}-\sum_{j=1}^{i} t_{n, j-1 \rightarrow j}\right)^{\left(1-\delta_{n, i \rightarrow i+1}\right)}\right], i=1,2,3
$$


When the information about $T_{n, i \rightarrow i+1}$ is represented by the possibility distribution $\mu_{\tilde{t}_{n, i \rightarrow i+1}}$, then the likelihood in Equation (11) reads (Denoeux, 2011):

$$
\tilde{L}(\vartheta \mid D)=\prod_{n=1}^{N}\left[\prod_{i=1}^{3} \tilde{f}_{T_{i \rightarrow i+1}}\left(t_{n, i \rightarrow i+1}\right)^{\delta_{n, i \rightarrow i+1}} \cdot R_{T_{i \rightarrow i+1}}\left(T_{m}-\sum_{j=1}^{i} t_{n, j-1 \rightarrow j}\right)^{\left(1-\delta_{n, i \rightarrow i+1}\right)}\right]
$$

where

$$
\tilde{f}_{T_{i \rightarrow i+1}}\left(t_{n, i \rightarrow i+1}\right)=\int_{0}^{+\infty} \mu_{\tilde{t}_{n, i \rightarrow i+1}}\left(t_{i \rightarrow i+1}\right) \cdot f_{T_{i \rightarrow i+1}}\left(t_{i \rightarrow i+1}\right) d t_{i \rightarrow i+1}=\int_{\underline{t}_{n, i \rightarrow i+1}}^{\bar{t}_{n, i \rightarrow i+1}} \mu_{\tilde{t}_{n, i \rightarrow i+1}}\left(t_{i \rightarrow i+1}\right) \cdot f_{T_{i \rightarrow i+1}}\left(t_{i \rightarrow i+1}\right) d t_{i \rightarrow i+1}
$$

That is, the imprecise evidence represented by the possibility distribution $\mu_{\tilde{t}_{n, i \rightarrow i+1}}\left(t_{i \rightarrow i+1}\right)$ forces to change $f_{T_{i \rightarrow i+1}}\left(t_{n, i \rightarrow i+1}\right)$ into $\tilde{f}_{T_{i \rightarrow i+1}}\left(t_{n, i \rightarrow i+1}\right)$ through Equation (15), which applies the Zadeh's definition of probability of a fuzzy event (Denoeux, 2011; Zadeh, 1996 ). Notice that $R_{T_{i \rightarrow i+1}}$ is not affected by fuzzy uncertainty as it relates to the case in which the transition has not been observed. The log-likelihood is given by:

$$
\tilde{\mathcal{L}}(\vartheta \mid D)=\log (\tilde{L}(\vartheta \mid D))=\tilde{\mathcal{L}}_{1 \rightarrow 2}\left(\alpha_{1}, \beta_{1} \mid D\right)+\tilde{\mathcal{L}}_{2 \rightarrow 3}\left(\alpha_{2}, \beta_{2} \mid D\right)+\tilde{\mathcal{L}}_{3 \rightarrow 4}\left(\alpha_{3}, \beta_{3} \mid D\right)
$$

where

$$
\tilde{\mathcal{L}}_{i \rightarrow i+1}\left(\vartheta_{i} \mid D\right)=\sum_{n=1}^{N} \log \left[\widetilde{f}_{T_{i \rightarrow i+1}}\left(t_{n, i \rightarrow i+1}\right)^{\delta_{n, i \rightarrow i+1}} \cdot R_{T_{i \rightarrow i+1}}\left(T_{m}-\sum_{j=1}^{i} t_{n, j-1 \rightarrow j}\right)^{\left(1-\delta_{n, i \rightarrow i+1}\right)}\right], i=1,2,3
$$

\subsection{Fuzzy Expectation-Maximization algorithm}

The EM algorithm has been proposed by (Dempster et al., 1977) as a broadly applicable and efficient mechanism for computing maximum likelihood estimates. It is made up of two steps (i.e., expectation and maximization), which are iteratively performed until the maximum of the likelihood function is achieved.

Inspired by the original EM algorithm, (Denoeux, 2011) has proposed an enhancement that extends its application to fuzzy evidence. We resort to this method for the maximization of each $\tilde{\mathcal{L}}_{i \rightarrow i+1}$ in Equation (17), $i=1,2,3$. 
Every iteration $q$ of the algorithm is composed by two steps:

STEP 1: Expectation step (E-step)

The expectation step requires the calculation of the expected value of the log-likelihood $\tilde{L}_{i \rightarrow i+1}$ conditional to a set of fuzzy evidences:

$$
Q\left(\vartheta_{i}, \vartheta_{i}^{q}\right)=E_{\vartheta_{i}^{q}}\left[\tilde{\mathcal{L}}_{i \rightarrow i+1} \mid D\right]
$$

which reads (Denoeux, 2011):

$$
Q\left(\vartheta_{i}, \vartheta_{i}^{q}\right)=\sum_{n=1}^{N}\left\{\frac{\int_{0}^{+\infty} \mu_{\tilde{t}_{n, i \rightarrow i+1}}\left(t_{i \rightarrow i+1}\right) \log [L(\vartheta \mid D)] L\left(\vartheta^{q} \mid D\right) d t_{i \rightarrow i+1}}{\int_{0}^{+\infty} \mu_{\tilde{t}_{n, i \rightarrow i+1}}\left(t_{i \rightarrow i+1}\right) L\left(\vartheta^{q} \mid D\right) d t_{i \rightarrow i+1}}\right\}
$$

Then, the expected value $Q\left(\vartheta_{i}, \vartheta_{i}^{q}\right)$ of $\tilde{\mathcal{L}}_{i \rightarrow i+1}$ in Equation (17) conditioned to the set of fuzzy evidences $D$, given the fit $\vartheta_{i}^{q}$ of $\vartheta_{i}$ at the current iteration $q$, becomes:

$$
\begin{aligned}
& Q\left(\vartheta_{i}, \vartheta_{i}^{q}\right)=
\end{aligned}
$$

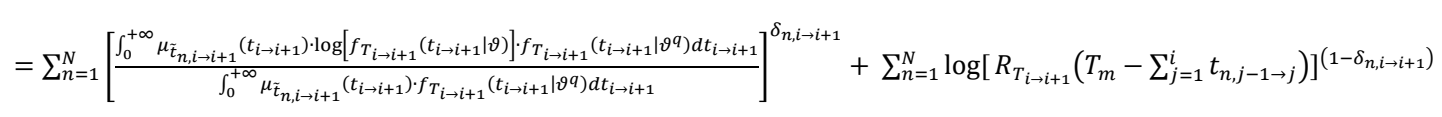

\section{STEP 2: Maximization step (M-step)}

The maximization step consists in maximizing $Q\left(\vartheta_{i}, \vartheta_{i}^{q}\right)$ with respect to $\vartheta_{i}$. To do this, we need to select an arbitrary guess vector $\theta^{q=0}$ which here is obtained by applying the standard MLE approach to the same dataset. The first iteration of the M-step stops when $\frac{\partial Q\left(\vartheta_{i}, \vartheta_{q}\right)}{\partial \vartheta_{i}}$ is smaller than an arbitrary fixed tolerance. The obtained parameters $\vartheta^{q=1}$, will be the guess vector for the following maximization step.

The expectation and maximization steps are iterated until the difference $\left|\tilde{\mathcal{L}}\left(\vartheta_{i}^{q+1}\right)-\tilde{\mathcal{L}}\left(\vartheta_{i}^{q}\right)\right|$ is smaller than some arbitrary fixed tolerance.

\section{Remaining Useful Life Estimation}


Once the parameters $\alpha_{i}$ and $\beta_{i}, i=1,2,3$, of the HCTSMM have been estimated, they can be used to estimate the PS RUL at any $k^{t h}$ inspection time, with $k=1, \ldots, M_{n}$. To highlight the benefits of including the expert knowledge in the model estimation parameters, we estimate the RUL in both the settings illustrated in Sections 3.1 and 3.2 (i.e., based only on data, and on data with additional imprecise information from the experts).

To this aim, we first consider the random variable $T_{i \rightarrow i+1}\left(t_{i \rightarrow i+1} \mid t_{n, i}^{0}\right)$, which represents the residual sojourn time in state $i$, provided that the component has already been in state $i$ for $t_{n, i}^{0}$ years. The PDF and CDF of $T_{i \rightarrow i+1}$ conditional on $t_{n, i}^{0}$ are indicated by $f_{T_{i \rightarrow i+1}}\left(t_{i \rightarrow i+1} \mid t_{n, i}^{0}\right)$ and $F_{T_{i \rightarrow i+1}}\left(t_{i \rightarrow i+1} \mid t_{n, i}^{0}\right)$, respectively. Accordingly, the expected value of the random variable $T_{i \rightarrow i+1}\left(t_{i \rightarrow i+1} \mid t_{n, i}^{0}\right)$, $E\left[T_{i \rightarrow i+1} \mid t_{n, i}^{0}\right]$ is

$$
E\left[T_{i \rightarrow i+1} \mid t_{n, i}^{0}\right]=\int_{t_{n, i}^{0}}^{+\infty}\left(t_{i \rightarrow i+1}-t_{n, i}^{0}\right) f_{T_{i \rightarrow i+1}}\left(t_{i \rightarrow i+1} \mid t_{n, i}^{0}\right) d t_{i \rightarrow i+1}=\left[\frac{E\left[T_{i \rightarrow i+1}\right]-\int_{0}^{t_{n, i}^{0}} t_{i \rightarrow i+1} \cdot f_{T_{i \rightarrow i+1}}\left(t_{i \rightarrow i+1}\right) d t_{i \rightarrow i+1}}{R_{T_{i \rightarrow i+1}}\left(t_{n, i}^{0}\right)}\right]-t_{n, i}^{0}
$$

where $E\left[T_{i \rightarrow i+1}\right]$ represents the expected value of $T_{i \rightarrow i+1}$.

The $\alpha$-quantile $q_{\alpha}\left[T_{i \rightarrow i+1} \mid t_{n, i}^{0}\right]$ of $T_{i \rightarrow i+1}\left(t_{i \rightarrow i+1} \mid t_{n, i}^{0}\right)$ can be derived from the inverse function $F_{T_{i \rightarrow i+1}}^{-1}\left(\alpha \mid t_{n, i}^{0}\right)$ of $F_{T_{i \rightarrow i+1}}\left(t_{i \rightarrow i+1} \mid t_{n, i}^{0}\right)$.

Suppose that at the inspection time $k \tau$, the component is found in state $i$; then, its RUL is the random variable $R U L(k \tau)$ defined as:

$$
R U L(k \tau)=T_{i \rightarrow i+1}\left(t_{i \rightarrow i+1} \mid t_{n, i}^{0}\right)+\sum_{j>i} T_{j \rightarrow j+1}, i, j \in\{1,2,3\}
$$

assuming that the sojourn time already spent in state $i$ is $t_{n, i}^{0}$.

The expected value, $E[R U L(k \tau)]$, and the $\alpha$-quantiles of $R U L(k \tau), q_{\alpha}[R U L(k \tau)]$, are given by, respectively: 


$$
E[R U L(k \tau)]= \begin{cases}\left\{\frac{E\left[T_{1 \rightarrow 2}\right]-\int_{0}^{t_{n, i}^{0}} t_{1 \rightarrow 2} \cdot f_{T_{1 \rightarrow 2}}\left(t_{1 \rightarrow 2}\right) d t_{1 \rightarrow 2}}{R_{T_{1 \rightarrow 2}}\left(t_{n, i}\right)^{0}}\right\}+E\left[T_{2 \rightarrow 3}\right]+E\left[T_{3 \rightarrow 4}\right]-k \tau, & \text { if } i=1 \\ \left\{\frac{E\left[T_{2 \rightarrow 3}\right]-\int_{0}^{t_{n, i}^{0}} t_{2 \rightarrow 3} \cdot f_{T_{2 \rightarrow 3}}\left(t_{2 \rightarrow 3}\right) d t_{2 \rightarrow 3}}{R_{T_{2 \rightarrow 3}}\left(t_{n, i}\right)^{0}}\right\}+E\left[T_{3 \rightarrow 4}\right]-t_{n, i}^{0}, & \text { if } i=2 \\ \left\{\frac{E\left[T_{3 \rightarrow 4}\right]-\int_{0}^{t_{n, i}} t_{3 \rightarrow 4} \cdot f_{T_{3 \rightarrow 4}}\left(t_{3 \rightarrow 4}\right) d t_{3 \rightarrow 4}}{R_{T_{3 \rightarrow 4}}\left(t_{n, i}^{0}\right)}\right\}-t_{n, i}^{0}, & \text { if } i=3\end{cases}
$$

$$
q_{\alpha}[R U L(k \tau)]: \int_{0}^{q_{\alpha}[R U L(k \tau)]} f_{R U L(k \tau)}(t) d t=\alpha
$$

where $f_{R U L(k \tau)}$ is the PDF of the random variable $R U L(k \tau)$.

The definition of $t_{n, i}^{0}$ depends on the case under investigation. In details, in the setting described in Section 3.1 with data only, we know that $t_{n, 1}^{0}=k \tau$ and $t_{n, i}^{0} \in\left[\left(k \tau-k_{n, i} \tau\right),\left(k \tau-\left(k_{n, i}-1\right) \tau\right)\right], i=$ 2,3. In this case, $E[R U L(k \tau)]$ and $q_{\alpha}[R U L(k \tau)]$ are interval estimates, whose lower and upper bounds are obtained by substituting $t_{n, i}^{0}$ with $\left(k \tau-k_{n, i} \tau\right)$ and $\left(k \tau-\left(k_{n, i}-1\right) \tau\right)$ (see Figure 3 ), respectively, in Equations (23) and (24).

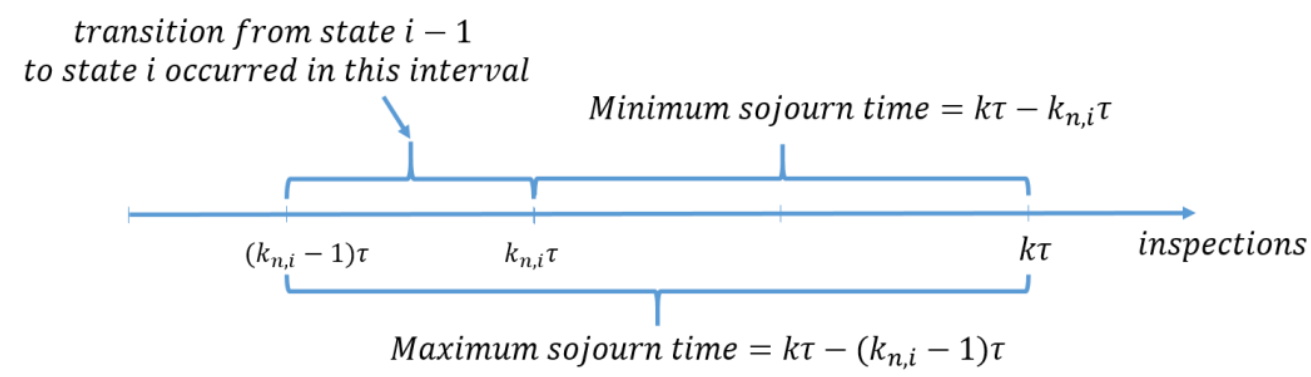

Figure 3: $t_{n, i}^{0}$ for setting in Section 3.1.

With respect to the setting illustrated in Section 3.2, the sojourn time in state $i$ corresponds to the difference between the crisp number $\left(k \tau-k_{n, i-1} \tau\right)$ and the triangular fuzzy number $\tilde{t}_{n, i-1 \rightarrow i}$, resulting in the possibility distribution $\mu_{\tilde{t}_{n, i}^{0}}\left(t_{n, i}^{0}\right)$ (Aven et al., 2014):

$$
\mu_{\tilde{t}_{n, i}^{0}}\left(t_{n, i}^{0}\right)=\left(\left(k \tau-k_{n, i-1} \tau-\bar{t}_{n, i \rightarrow i+1}\right),\left(k \tau-k_{n, i-1} \tau-\tilde{t}_{n, i \rightarrow i+1}\right),\left(k \tau-k_{n, i-1} \tau-\underline{t}_{n, i \rightarrow i+1}\right)\right)
$$


which is shown in Figure 4.

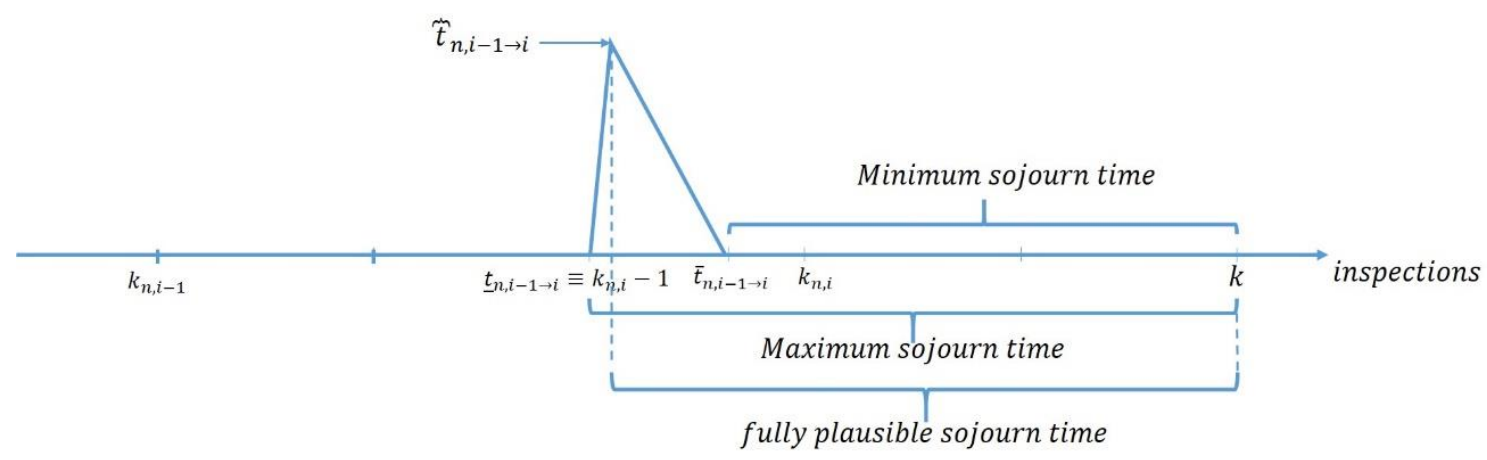

Figure 4: $\boldsymbol{t}_{n, i}^{0}$ for setting in Section 3.2.

Since the random variable $R U L(k \tau)$ depends on the sojourn time, as shown in Equation (22), it is affected by the epistemic uncertainty on $t_{n i}^{0}$, and, thus, in the case considered it becomes a fuzzy number, described by a possibility distribution. Therefore, the expected value and the $\alpha$-quantile estimates of the fuzzy $R U L(k \tau)$, according to the Zadeh extension principle, are fuzzy numbers too (Zadeh, 1996; Aven et al., 2014). The lower bound, core and upper bound of the estimates of $E[R U L(k \tau)]$ and $q_{\alpha}[R U L(k \tau)]$ are obtained by combining Equation (25) with Equation (23) and Equation (24), respectively.

Finally, notice that if at inspection time $k \tau$ the component is found in state 1 (i.e., no transition has been observed), then $E[R U L(k \tau)]$ and $q_{\alpha}[R U L(k \tau)]$ do not depend on $t_{n, i}^{0}$.

\section{CASE STUDY}

In this Section, the settings described in Sections 3.1 and 3.2 and the RUL estimation procedure discussed in Section 4 are applied to a simulated case study concerning the 4-states PS degradation process described in Section 2.

\subsection{Data Simulation}

We have artificially generated $\mathrm{N}=100$ degradation paths by Monte-Carlo (MC) sampling from the Markov Model described in (Fleming, 2004), which assumes that the transition times are exponentially distributed with scale parameters $\lambda_{i \rightarrow i+1}$ given in Table 1 .

\begin{tabular}{|c|c|}
\hline$i$ & $\lambda_{i \rightarrow i+1}\left(y^{-1}\right)$ \\
\hline 1 & $4.35 \times 10^{-4}$ \\
\hline 2 & $1.79 \times 10^{-4}$ \\
\hline
\end{tabular}




\begin{tabular}{|l|l|}
\hline 3 & $1.97 \times 10^{-2}$ \\
\hline
\end{tabular}

Table 1: Values of the scale parameter $\lambda_{i \rightarrow i+1}$ used to simulate the degradation process (Fleming, 2004).

Then, a right censoring mechanism has been applied to the gathered data, with $T_{m}=60$ years. This value is taken from (Di Maio et al., 2015) and considers that typically NPPs have a life time of 40 years, plus possible extension. We consider that the system is periodically inspected with period $\tau=$ 5 years.

The case study dataset is summarized in Table 2a, which reports for every state $i$ (first column) the corresponding number of components that have entered in state $i$ (second column) and the number of components which are found in state $i$ at inspection $k_{n, i}=M_{n}$ (third column).

Table 2a:

\begin{tabular}{|c|c|c|}
\hline$i$ & $\begin{array}{c}\text { Number of components } \\
\text { entered in state } i\end{array}$ & $\begin{array}{c}\text { Number of components found in state } i \text { at } \\
\text { inspection } M_{n}\end{array}$ \\
\hline 1 & 100 & 94 \\
\hline 2 & 6 & 4 \\
\hline 3 & 2 & 1 \\
\hline 4 & 1 & 1 \\
\hline
\end{tabular}

dataset.

Case study

To better understand the case study dataset, the degradation paths of the six components that entered a state $i>1$ are summarized in Table $2 \mathrm{~b}$, where the first column reports the $n^{\text {th }}$ component, whereas the other three columns report the values of $k_{n, i}, i=2,3,4$, respectively, as defined in Subsection 2.1.

Table 2b: Case study

\begin{tabular}{|c|c|c|c|}
\hline$n$ & $k_{n}, 2$ & $k_{n, 3}$ & $k_{n, 4}$ \\
\hline 4 & 4 & - & - \\
\hline 13 & 11 & - & - \\
\hline 17 & 11 & - & - \\
\hline 41 & 8 & 9 & - \\
\hline 74 & 3 & - & 12 \\
\hline 91 & 1 & 10 & - \\
\hline
\end{tabular}

dataset. 
Obviously, in this simulated case study we do not have real expert judgments. Nevertheless, for a better understanding of how the expert knowledge can influence the estimation of the unknown parameters, we investigate three different settings relating to as many risk attitudes of the expert. Namely, we consider three different types of possibility distributions $\mu_{\tilde{t}_{n, i \rightarrow i+1}}\left(t_{n, i \rightarrow i+1}\right)$ corresponding to the information about the transition time retrievable from a moderately risk-averse (Figure 5), risk- averse (Figure 6), and risk- prone (Figure 7) expert. For brevity, these three settings will be referred to as cases $C_{1}, C_{2}$ and $C_{3}$, respectively, whereas the MLE setting will be referred to as $C_{0}$.

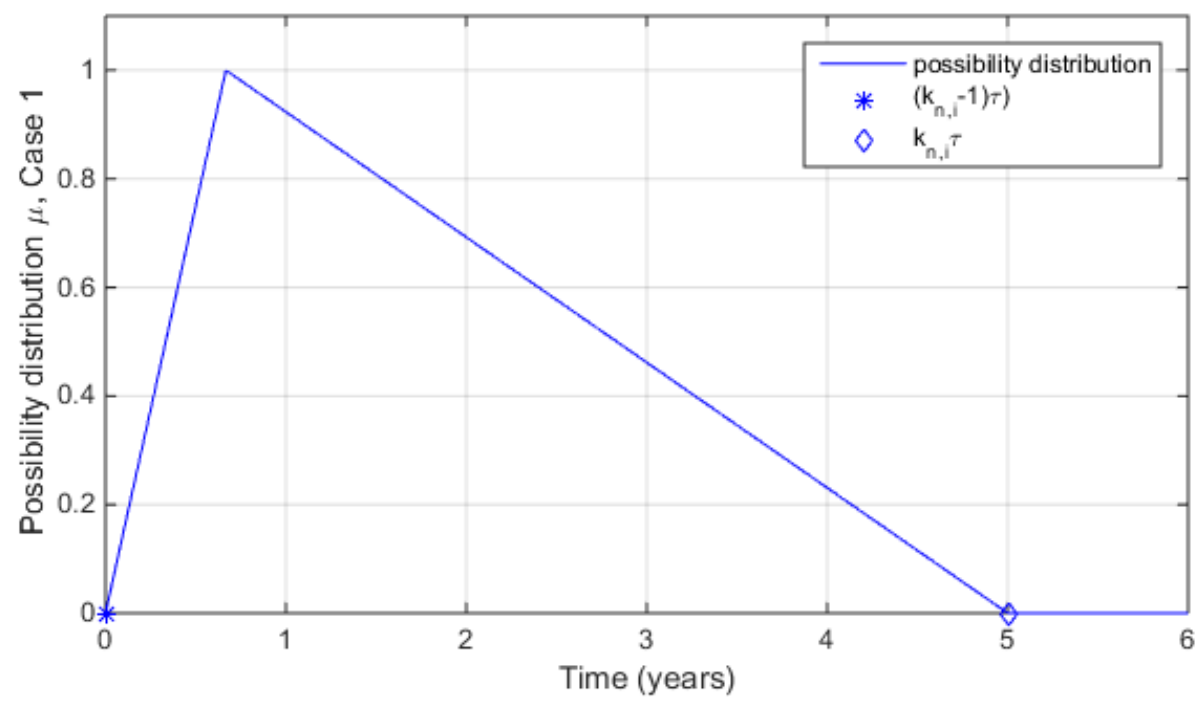

Figure 5: Example of possibility distribution of case $C_{1}$ (moderately risk-averse).

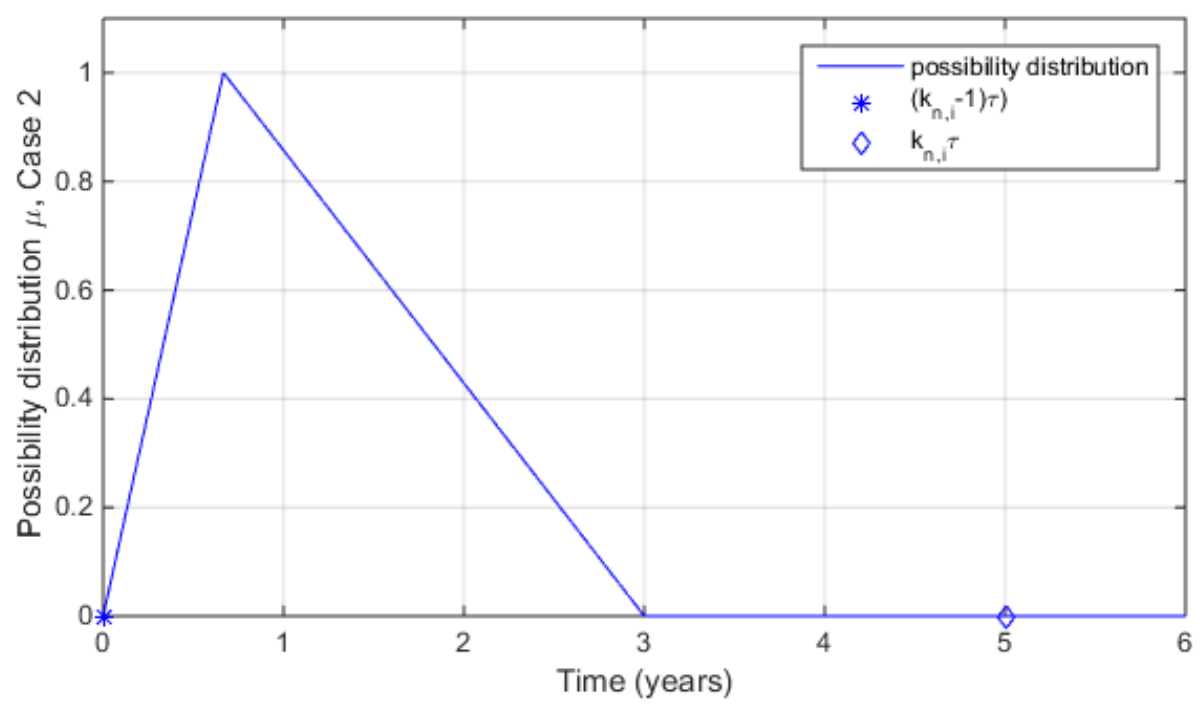

Figure 6: Example of possibility distribution of case $C_{2}$ (risk-averse). 


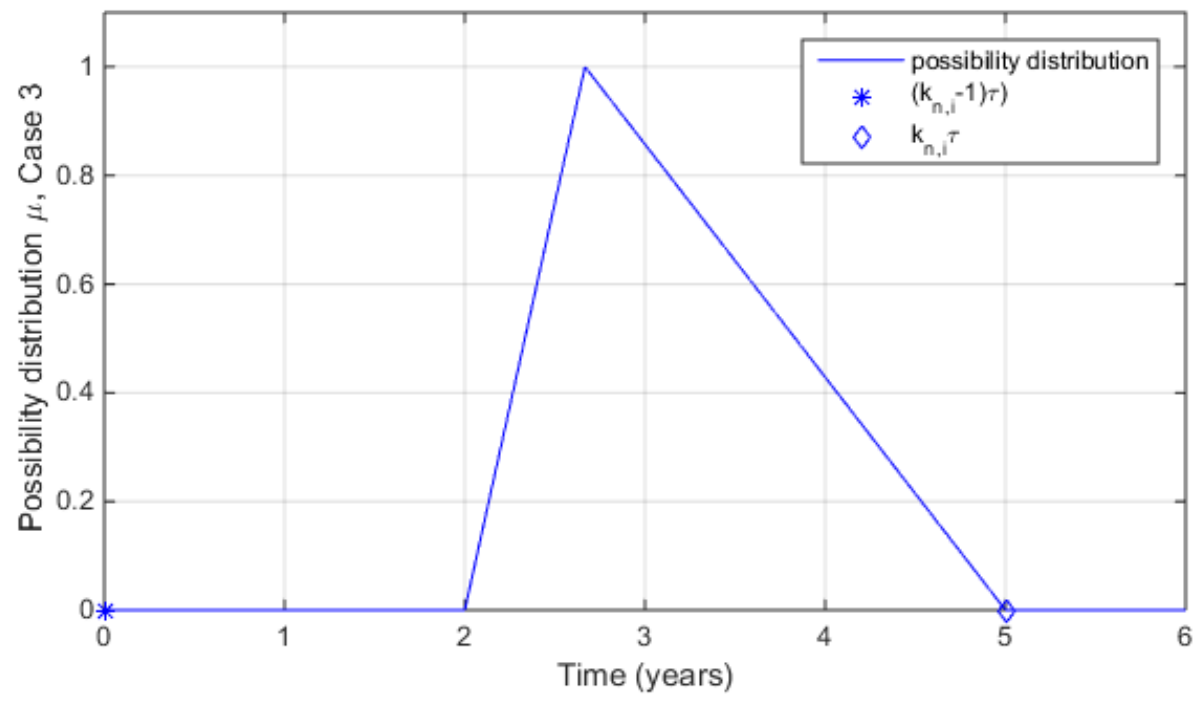

Figure 7: Example of possibility distribution of case $C_{3}$ (risk-prone).

In the first case, the expert does not commit her/him-self and, thus, does not reduce the support of the possibility distribution; she/he only gives the fully plausible value of $t_{n, i \rightarrow i+1}$, within the interval $\left[\left(k_{n, i+1}-1\right) \tau ; k_{n, i+1} \tau\right]$, but closer to its lower bound; namely, he/she sets $\tilde{t}_{n, i \rightarrow i+1}=0.667+\dot{t}_{n, i}^{0}$, where $\dot{t}_{n, i}^{0}=\left(k_{n, i+1}-1\right) \tau-k_{n, i} \tau$ (i.e., the time elapsed from the first inspection time in which the component has been found in state $i$ and the last one). In the second case, the expert is more conservative, i.e., he/she feels that the transition surely lies in the interval $\left[\left(k_{n, i+1}-1\right) \tau ;\left(k_{n, i+1} \tau-\right.\right.$ $2)$ ], with core $\dddot{t}_{n, i \rightarrow i+1}=0.667+\dot{t}_{n, i}^{0}$. Finally, the case in Figure 7 refers to an expert that feels confident on the system and, thus, states that the transition surely occurred in the interval $\left.\left[\left(k_{n, i+1}-1\right) \tau+2\right) ; k_{n, i+1} \tau\right]$, the fully plausible value $\tilde{t}_{n, i \rightarrow i+1}=2.667+\dot{t}_{n, i}^{0}$.

\subsection{Parameters Estimation results}

Tables 3-6 report the results obtained for cases $C_{0}-C_{3}$, respectively, which are summarized by the expected value, $E\left[T_{i \rightarrow i+1}\right]$, the variance, $\operatorname{Var}\left[T_{i \rightarrow i+1}\right]$, the median, $q_{0.5}\left[T_{i \rightarrow i+1}\right]$ and the interval $I=$ $\left[q_{0.05}\left[T_{i \rightarrow i+1}\right] ; q_{0.95}\left[T_{i \rightarrow i+1}\right]\right]$ which covers $90 \%$ of the values of the random variables $T_{i \rightarrow i+1}, i=$ $1,2,3$, obeying Weibull distributions.

\begin{tabular}{|c|c|c|c|c|c|}
\hline \multicolumn{6}{|l|}{ MLE estimation } \\
\hline $\boldsymbol{i}$ & $\widehat{\boldsymbol{\vartheta}}_{\mathrm{MLE}}$ & $\operatorname{Var}\left\{\boldsymbol{T}_{i \rightarrow i+1}\right\}$ & $E\left\{\boldsymbol{T}_{i \rightarrow i+1}\right\}$ & $\boldsymbol{q}_{0.5}\left\{\boldsymbol{T}_{i \rightarrow i+1}\right\}$ & I \\
\hline
\end{tabular}




\begin{tabular}{|l|l|l|l|l|l|}
\hline 1 & $\begin{array}{l}\alpha_{1}=1.494 \mathrm{e}+03 \\
\beta_{1}=0.866\end{array}$ & $3.466 \mathrm{e}+06$ & $1.607 \mathrm{e}+03$ & 979.22 & {$[50.15 ; 5319.51]$} \\
\hline 2 & $\begin{array}{l}\alpha_{2}=104.41 \\
\beta_{2}=0.65\end{array}$ & $5.097 \mathrm{e}+04$ & 142.13 & 59.52 & {$[1.07 ; 561.88]$} \\
\hline 3 & $\begin{array}{l}\alpha_{3}=21.29 \\
\beta_{3}=1.44\end{array}$ & 185.04 & 19.32 & 16.52 & {$[2.71 ; 45.62]$} \\
\hline
\end{tabular}

Table 3: Results for case $C_{0}$.

\begin{tabular}{|c|c|c|c|c|c|}
\hline \multicolumn{6}{|c|}{ FEM estimation } \\
\hline $\bar{i}$ & $\widehat{\boldsymbol{\vartheta}}_{\text {FEM }}$ & $\operatorname{Var}\left\{T_{i \rightarrow i+1}\right\}$ & $E\left\{T_{i \rightarrow i+1}\right\}$ & $q_{0.5}\left\{T_{i \rightarrow i+1}\right\}$ & I \\
\hline 1 & $\begin{array}{l}\alpha_{1}=1.671 \mathrm{e}+03 \\
\beta_{1}=0.837\end{array}$ & $4.858 \mathrm{e}+07$ & $1.835 \mathrm{e}+03$ & 1078.75 & {$[47.48 ; 6239.39]$} \\
\hline 2 & $\begin{array}{l}\alpha_{2}=98.26 \\
\beta_{2}=0.68\end{array}$ & $3.698 \mathrm{e}+04$ & 127.60 & 57.41 & {$[1.28 ; 484.85]$} \\
\hline 3 & $\begin{array}{l}\alpha_{3}=21.57 \\
\beta_{3}=1.32\end{array}$ & 229.89 & 19.85 & 16.34 & {$[2.23 ; 49.47]$} \\
\hline
\end{tabular}

Table 4: Results for case $C_{1}$.

The parameters values estimated in case $C_{1}$ are similar to those in case $C_{0}$. This is due to the fact that the simulated dataset is composed by $94 \%$ of components that have never experienced any transition during the mission time i.e., they are still in state 1 at $t=T_{m}$ (Table 2). For these components, the expert opinion on state transition times is not exploited. Moreover, in case $C_{1}$, the expert gives a possibility distribution whose support is coincident with the interval between successive inspections, which corresponds to the interval-censored data considered in case $C_{0}$.

\begin{tabular}{|c|c|c|c|c|c|}
\hline FEM est & & & & & \\
\hline $\bar{i}$ & $\widehat{\boldsymbol{\vartheta}}_{\text {FEM }}$ & $\operatorname{Var}\left\{T_{i \rightarrow i+1}\right\}$ & $E\left\{T_{i \rightarrow i+1}\right\}$ & $q_{0.5}\left\{T_{i \rightarrow i+1}\right\}$ & I \\
\hline 1 & $\begin{array}{l}\alpha_{1}=2.134 \mathrm{e}+03 \\
\beta_{1}=0.779\end{array}$ & $1.020 \mathrm{e}+06$ & $2.464 \mathrm{e}+03$ & 1334.30 & {$[47.71 ; 8753.99]$} \\
\hline
\end{tabular}




\begin{tabular}{|l|l|l|l|l|l|}
\hline 2 & $\begin{array}{l}\alpha_{2}=108.69 \\
\beta_{2}=0.61\end{array}$ & $7.018 \mathrm{e}+04$ & 156.91 & 60.18 & {$[0.92 ; 640.69]$} \\
\hline 3 & $\begin{array}{l}\alpha_{3}=21.90 \\
\beta_{3}=1.20\end{array}$ & 293.80 & 20.57 & 16.16 & {$[1.87 ; 54.36]$} \\
\hline
\end{tabular}

Table 5: Results for case $C_{2}$.

\begin{tabular}{|c|l|l|l|l|l|}
\hline \multicolumn{1}{|c|}{ EEM estimation } & \multicolumn{1}{|c|}{$\widehat{\boldsymbol{\vartheta}}_{\text {FEM }}$} & \multicolumn{1}{|c|}{$\operatorname{Var}\left\{\boldsymbol{T}_{\boldsymbol{i} \rightarrow \boldsymbol{i + 1}}\right\}$} & \multicolumn{1}{|c|}{$\boldsymbol{E}\left\{\boldsymbol{T}_{\boldsymbol{i} \rightarrow \boldsymbol{i + 1}}\right\}$} & $\boldsymbol{q}_{\mathbf{0 . 5}}\left\{\boldsymbol{T}_{\boldsymbol{i} \rightarrow \boldsymbol{i + 1}}\right\}$ & I \\
\hline $\boldsymbol{i}$ & $\begin{array}{l}\alpha_{1}=1.011 \mathrm{e}+03 \\
\beta_{1}=0.985\end{array}$ & $1.066 \mathrm{e}+07$ & $1.017 \mathrm{e}+03$ & 697.54 & {$[50.66 ; 3085.01]$} \\
\hline 2 & $\begin{array}{l}\alpha_{2}=77.92 \\
\beta_{2}=0.89\end{array}$ & $8.427 \mathrm{e}+03$ & 82.16 & 51.78 & {$[2.86 ; 265.42]$} \\
\hline 3 & $\alpha_{3}=20.73$ & 133.69 & 18.54 & 16.59 & {$[3.38 ; 40.21]$} \\
\hline
\end{tabular}

Table 6: Results for case $C_{3}$.

The impact of including the expert information is appreciable when case $C_{0}$ is compared with cases $C_{2}$ and $C_{3}$, where the supports of the possibility distributions on the transition times do not coincide with the whole interval $\left[\left(k_{n, i+1}-1\right) \tau ; k_{n, i+1} \tau\right]$. We consider only the transitions observed before $T_{m}$, so that the parameter estimation is influenced by transition times $t_{n, i \rightarrow i+1}<60$ for which the expert expresses his/her opinion. The influence of the expert risk-attitude can be appreciated when comparing the estimated parameters $\beta$. In particular, for the first and the second transition, $\beta_{1}$ and $\beta_{2}$ are smaller than 1 , which means decreasing estimated transition rate over time, but, in case $C_{3}, \beta_{1}$ and $\beta_{2}$ are larger than the estimates of cases $C_{0}$ and $C_{2}$.

In order to clarify the influence of the expert risk-attitude on transition rates, we have computed $\lambda_{C 0}$ for case $C_{0}$ (i.e., the expert is moderately risk-averse), $\lambda_{C 2}$ for case $C_{2}$ (i.e., the expert is risk-averse), and $\lambda_{C 3}$ for case $C_{3}$ (i.e, the expert is moderately risk-prone), according to the expression for $\lambda_{i}$ given in Equation (4). The behaviors over time of the estimated transition rates for the transition from state 1 to state 2 are shown in Figure 8: in the initial part of the time axis, the transition rate related to the risk-prone expert is smaller than those of the moderately risk-averse and risk-averse experts. 


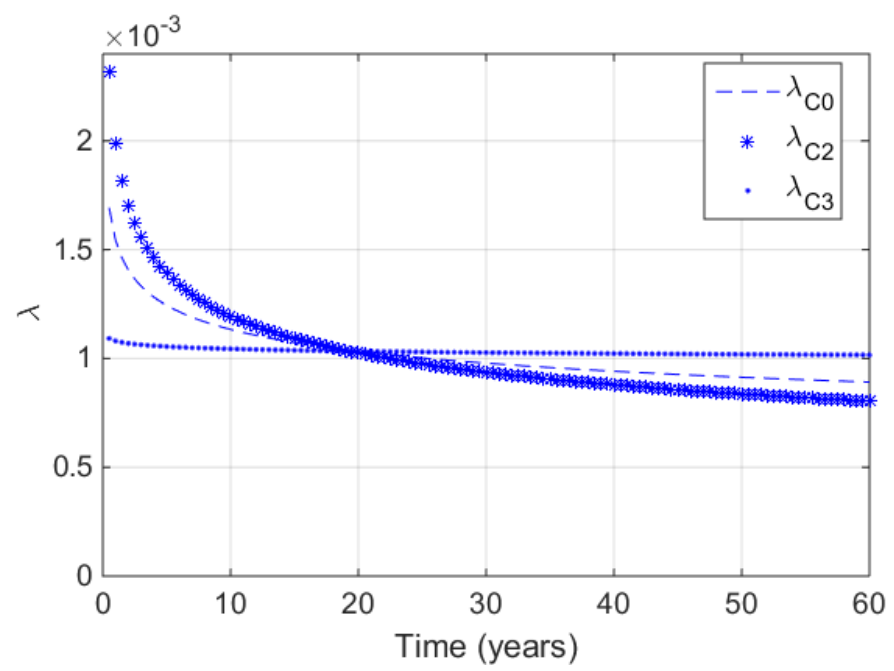

Figure 8: Transition rates for transition from state 1 to state 2, with $\lambda_{C_{0}}$ for Case $C_{0}, \lambda_{C_{2}}$ for Case $C_{2}$ and $\lambda_{C_{3}}$ for Case $C_{3}$.

Similarly, the rate for the third transition estimated in case $C_{3}$ is initially larger than those in case $C_{0}$ and $C_{2}$, as shown in Figure 9.

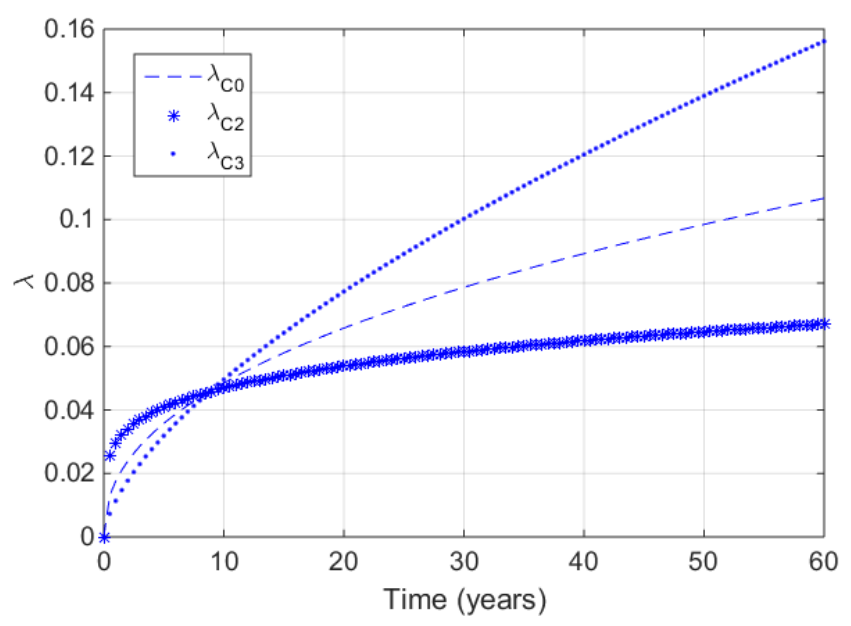

Figure 9: Transition rates $\lambda_{c 0}, \lambda_{c 2}$ and $\lambda_{c 3}$ for transition from state 3 to state. 
Figure 10 shows the estimated reliability function for the different scenarios, which has been derived by the Monte Carlo (MC) approach summarized in Appendix A.

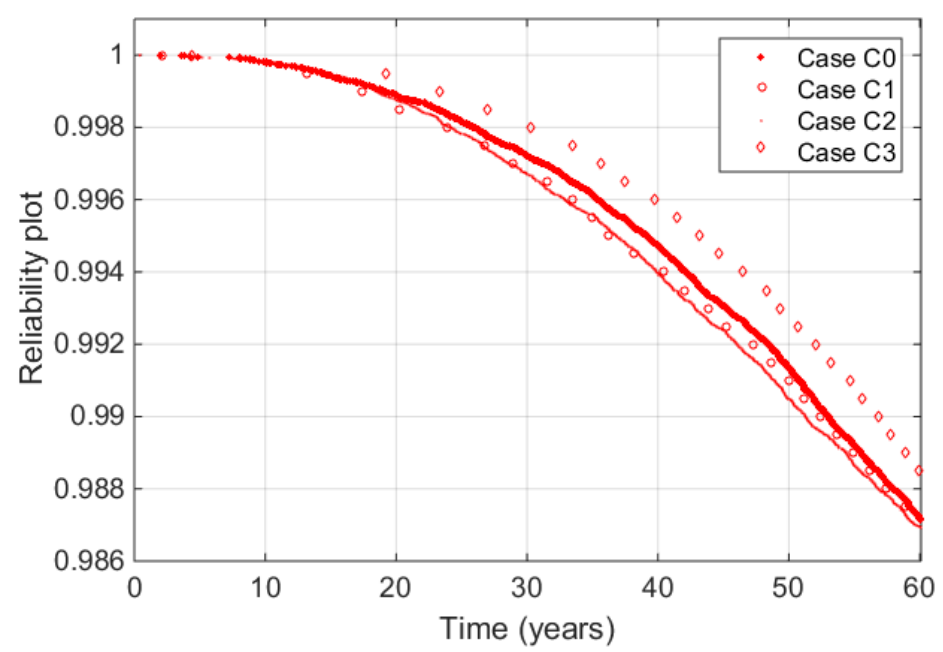

Figure 10: Estimated reliability function.

\subsection{RUL estimation results}

In this Section, we report the results of the estimation of $E[R U L(k \tau)]$ and $q_{\alpha}[R U L(k \tau)]$, by applying the procedure described in Section 4.

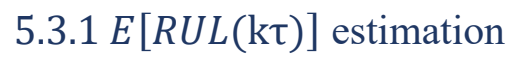

The expected value $E[R U L((k \tau))]$ of the random variable $R U L(k \tau)$ has been estimated according to Equation (23) at each inspection time $k \tau, k=1 \ldots M_{n}$, assuming that at this time instant the component can be found in state 1 , state 2 or state 3.

Figures 11-16 show the evolution of $E[R U L(k \tau)]$ over $k \tau$. We firstly compare the results obtained in case $C_{0}$ with those obtained in case $C_{1}$, as their estimated model parameters are similar to each other. Then, RUL estimates in case $C_{0}$ are compared to those of cases $C_{2}$ and $C_{3}$. For visualization, the axes of these Figures have different scales; yet, for states $i=2,3$ the $E[R U L(k \tau)]$ is divided into two plots: a) where $k \tau=\{5, \ldots, 30\}$ and b) where $k \tau=\{35, \ldots, 60\}$. The estimates of $E[R U L(k \tau)]$, given that the PS is in state 1, are reported in Figure 11, both for case $C_{1}$ and for case $C_{0}$. Since no transition has been observed when the component is found in state 1 , then the estimates do not depend on $t_{n, i}^{0}$ and are not affected by uncertainty. The difference between the estimated RULs is only due to the different values of $\vartheta$. 


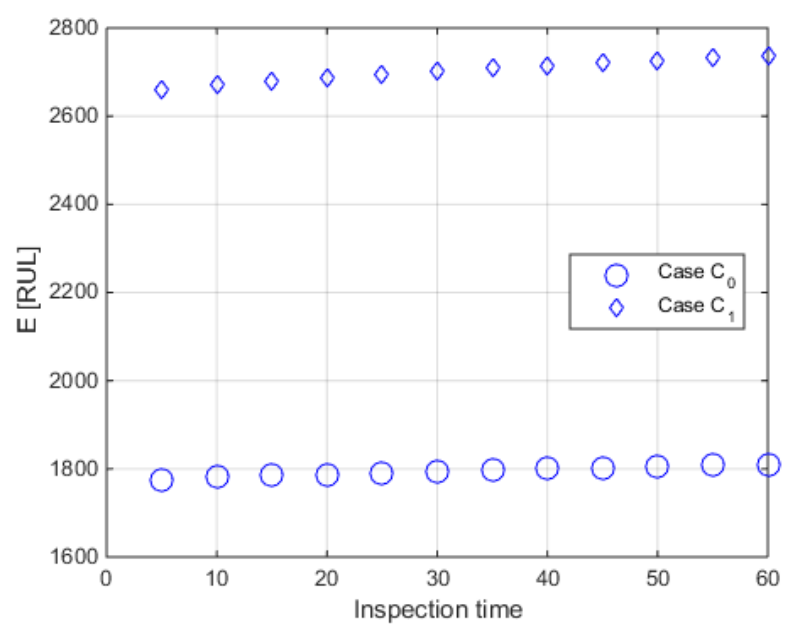

Figure 11: Results for Cases $C_{0}$ and $C_{1}$; state 1; E[RUL] and Inspection time in years.

In Figures 12-13, the estimates of $E[R U L(k \tau)]$ for states 2 and 3 are shown, respectively, which are intervals for case $C_{0}$ and triangular possibility distributions for case $C_{1}$. From the comparison of these Figures, we can notice that in case $C_{1}$ the uncertainty on $E[R U L((k \tau))]$ is always smaller than that in case $C_{0}$, at any inspection time. This is due to the fact that the expert statements introduce an additional information on the system behaviour, which allows better specifying the value of $E[R U L(k \tau)]$ in the interval containing its unknown value. This result highlights the contribution of the method here proposed to exploit any source of information that can corroborate data.
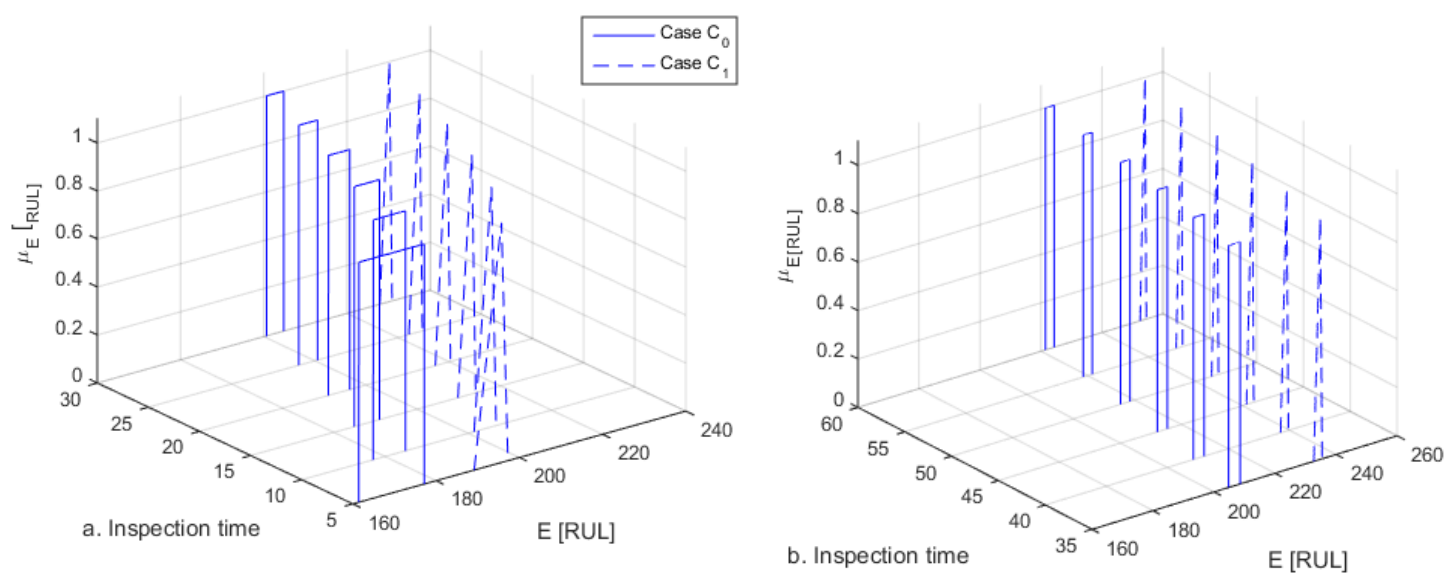

Figure 12: Results for cases $C_{0}$ and $C_{1}$; state 2; E[RUL] and Inspection time in years. 

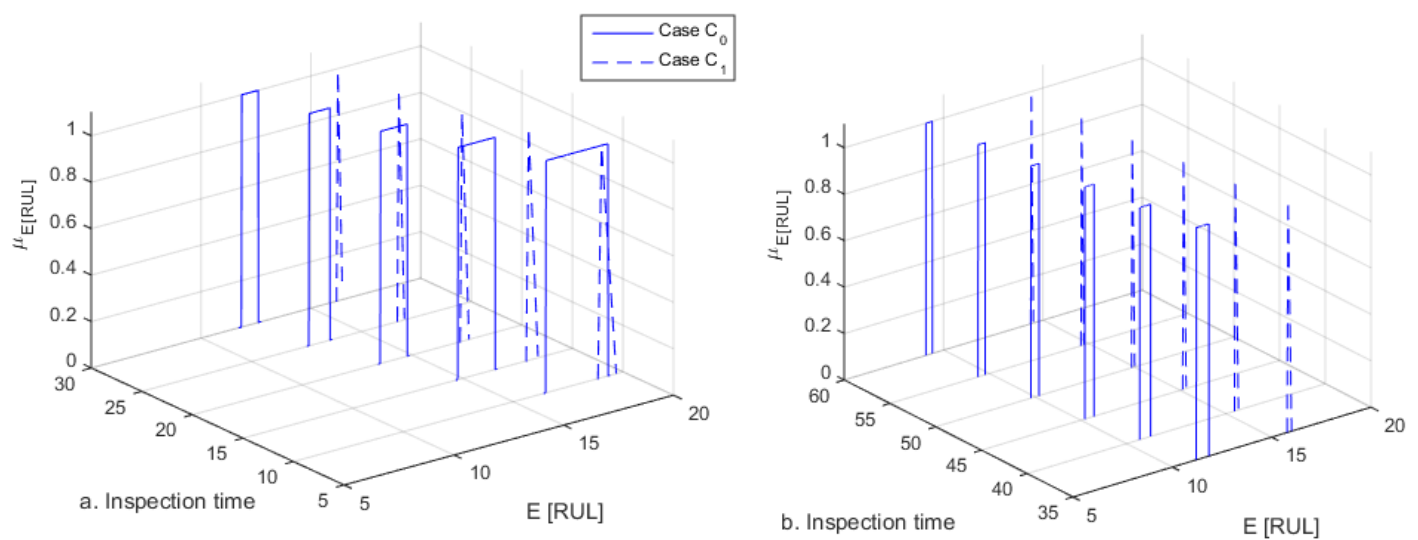

Figure 13: Results for cases $C_{0}$ and $C_{1}$; state 3 ; E[RUL] and Inspection time in years.

With respect to the comparison of case $C_{0}$ to cases $C_{2}$ and $C_{3}$, Figure 14 compares the $E[R U L(k \tau)]$ estimates when the component is in state 1 , which do not depend on $t_{n, i}^{0}$. The estimates are not affected by uncertainty, as introduced previously, and the ones of case $C_{0}$ are always smaller than those of case $C_{2}$ and larger than those of case $C_{3}$. This difference reflects the different expected value estimated in each case, in fact the expected value of case $C_{2}$ is larger than the others, while the one of case $C_{3}$ is smaller.

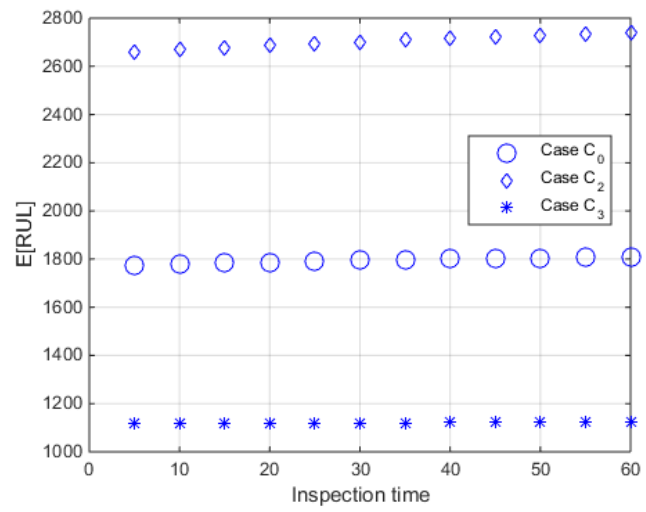

Figure 14: Results for cases $C_{0}, C_{2}$ and $C_{3}$; state 1; E[RUL] and Inspection time in years.

Figure 15 compares the results of case $C_{0}$ to those of cases $C_{2}$ and $C_{3}$, when the PS is found in state 2. We can note that intervals estimated in case $C_{0}$ never contain the fuzzy estimates of cases $C_{2}$ and $C_{3}$.
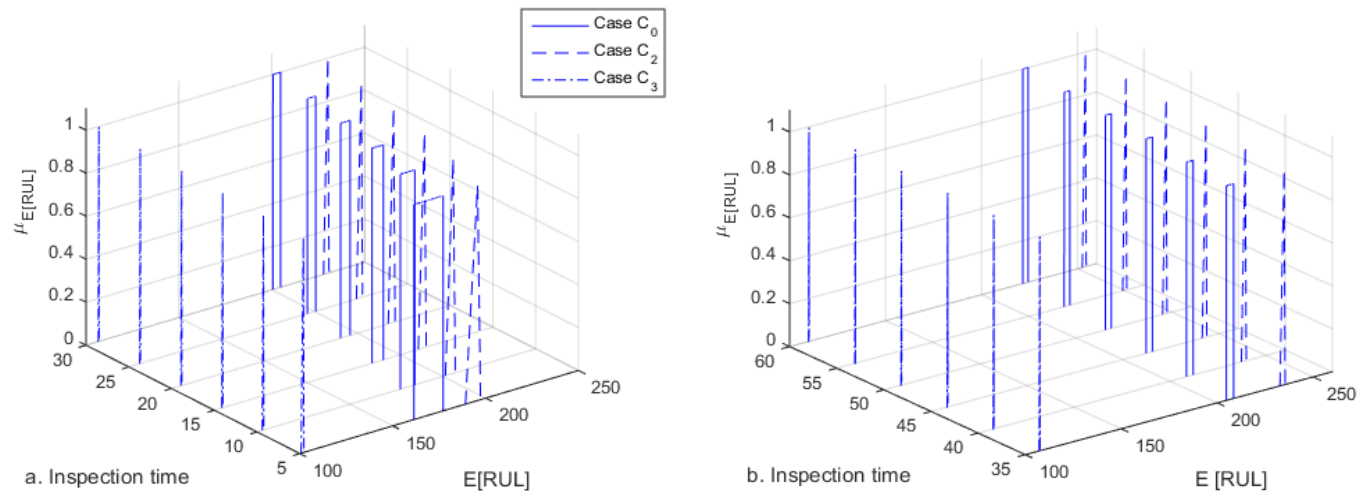
Figure 15: Results for cases $C_{0}, C_{2}$ and $C_{3}$; state 2; E[RUL] and Inspection time in years.

In particular, the estimates in case $C_{3}$ are always smaller than the corresponding ones of case $C_{0}$, which are smaller than those of case $C_{2}$. This is due to the fact that the estimates of $E\left[T_{i \rightarrow i+1}\right], i=$ $1,2,3$, are larger in case $C_{2}$ than in case $C_{0}$, while they are larger in case $C_{0}$ than in case $C_{3}$. Figure 16 shows the RUL estimates in cases $C_{0}, C_{2}$ and $C_{3}$ assuming that the component is found in state 3. In this case, the estimates are closer to each other since the corresponding estimated parameters $\vartheta$ for the third transition are similar to each other, as underlined previously.
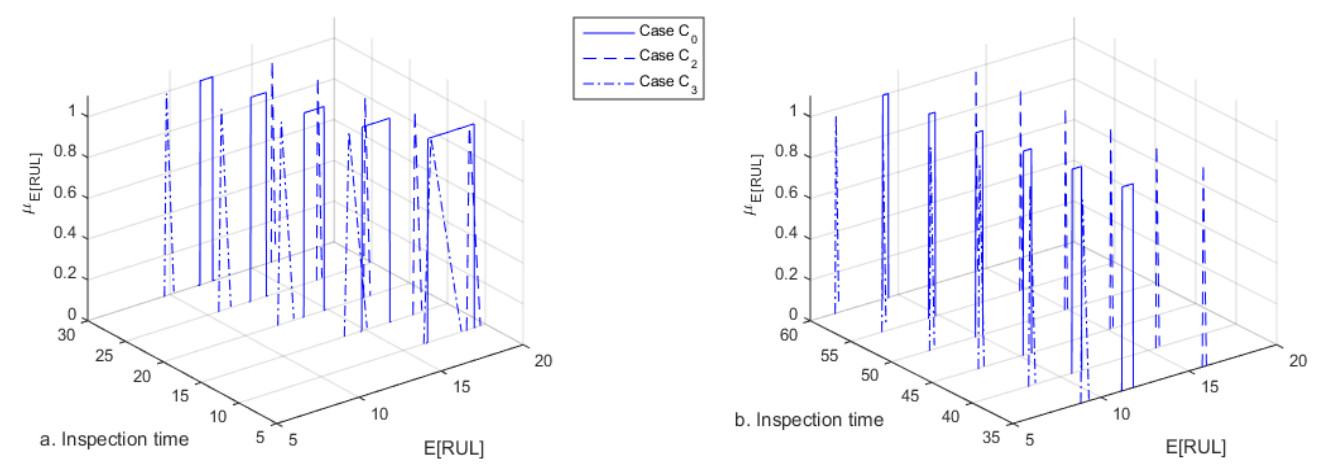

Figure 16: Results for cases $C_{0}, C_{2}$ and $C_{3}$; state $3 ; \mathrm{E}[\mathrm{RUL}]$ and Inspection time in years.

\subsection{2 $\boldsymbol{q}_{\alpha}[\boldsymbol{R} \boldsymbol{U} \boldsymbol{L}(\boldsymbol{k \tau})]$ estimation}

"The interval containing $\alpha \%$ of the values of the random variable $R U L(k \tau)$, Equation (24), has been estimated through MC simulation (see Appendix B), at each inspection time $k \tau, k=1 \ldots M_{n}$, assuming that at this time instant the component can be found in state 1, state 2 or state 3 .

Figures 17-22 show the evolution $q_{\alpha}[R U L(k \tau)]$ over $k \tau$ and we report the estimates for $\alpha=0.1$. We firstly compare the results obtained in case $C_{0}$ with those obtained in case $C_{1}$, as their estimated model parameters are similar to each other. Then RUL estimates in Case $C_{0}$ are compared to those of cases $C_{2}$ and $C_{3}$. For visualization, the axes of these Figures have different scales; yet, for states $i=2,3$ the $q_{\alpha}[R U L(k \tau)]$ is divided into two plots: a) where $k \tau=\{5, \ldots, 30\}$ and b) where $k \tau=\{35, \ldots, 60\}$. The estimates of $q_{\alpha}[R U L(k \tau)]$, given that the PS is in state 1, are reported in Figure 15, both for case $C_{1}$ and for case $C_{0}$. Since no transition has been observed when the component is found in state 1 , then the estimates do not depend on $t_{n, i}^{0}$ and are not affected by uncertainty. 


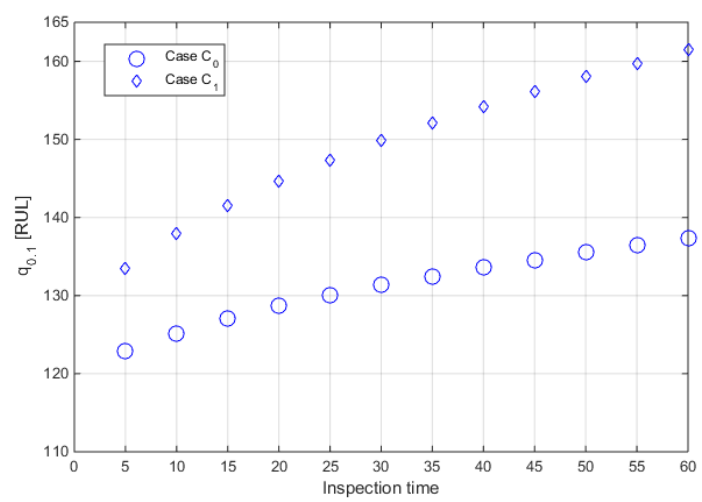

Figure 17: Results for case $C_{0}, C_{1}$, state $1 ; q_{0.1}[R U L]$ and Inspection time in years.

In Figures 18-19, the estimates are shown for the states 2 and 3, respectively, which are intervals for case $C_{0}$ and traingular possibility distributions for case $C_{1}$. From the comparison of these Figures, we can notice that in case $C_{1}$ the uncertainty on $q_{\alpha}[R U L(k \tau)]$ is always smaller than that in case $C_{0}$, at any inspection time. This is thanks to the additional information given by the expert and exploited in the estimation, which allows better specifying the value of $q_{\alpha}[R U L(k \tau)]$ in the interval containing its unknown value.
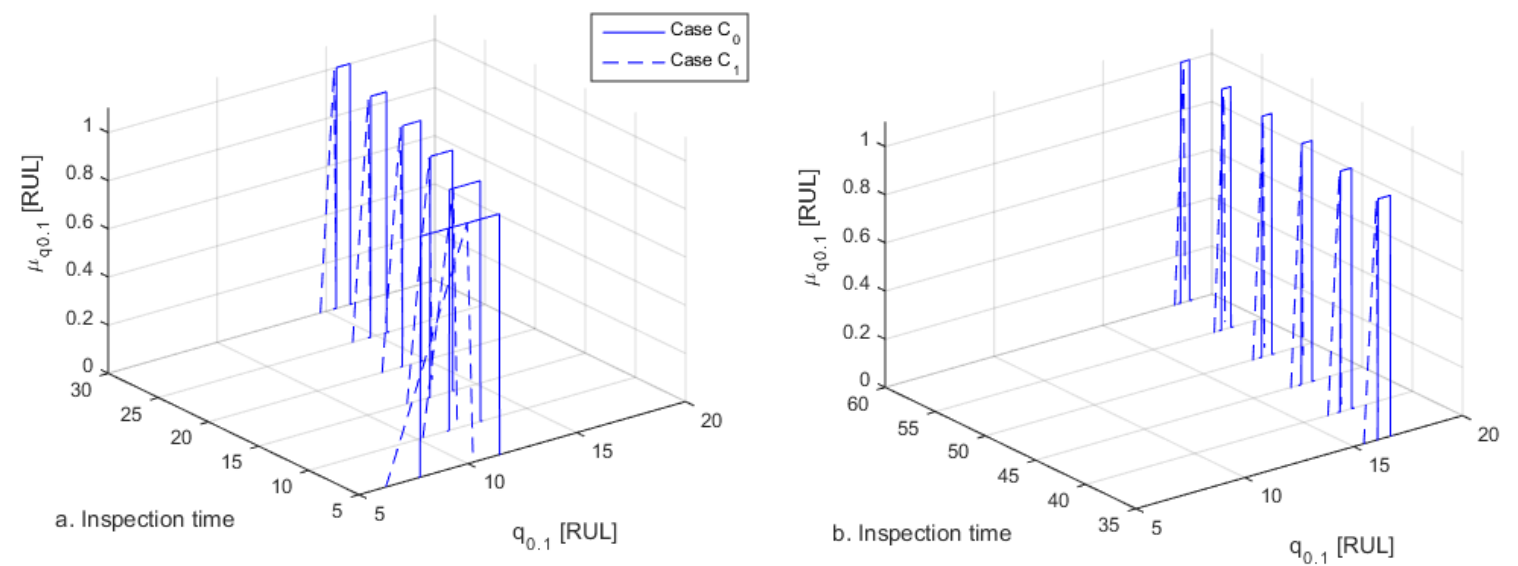

Figure 18: Results for case $C_{0}, C_{1}$, state $2 ; q_{0.1}[R U L]$ and Inspection time in years.
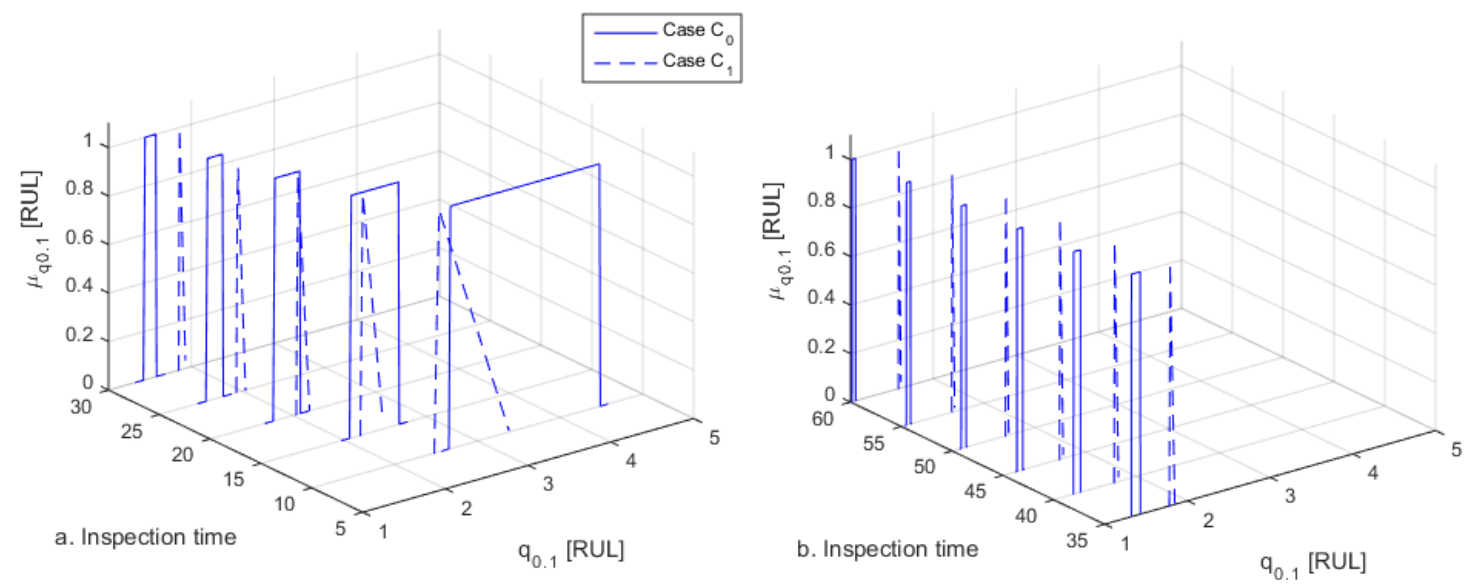

Figure 19: Results for case $C_{0}, C_{1}$, state $3 ; q_{0.1}[R U L]$ and Inspection time in years. 
With respect to the comparison of case $C_{0}$ to cases $C_{2}$ and $C_{3}$, Figure 20 compares the $q_{\alpha}[R U L(k \tau)]$ estimates when the component is in state 1 . The estimates of case $C_{2}$ are larger than the others, throughout the time range, reflecting the larger expected values estimated for the transition times, in this case. Whereas, the estimates of case $C_{3}$ are smaller than the others, over all the time range, reflecting the smaller expected values estimated for the transition times, in this case.

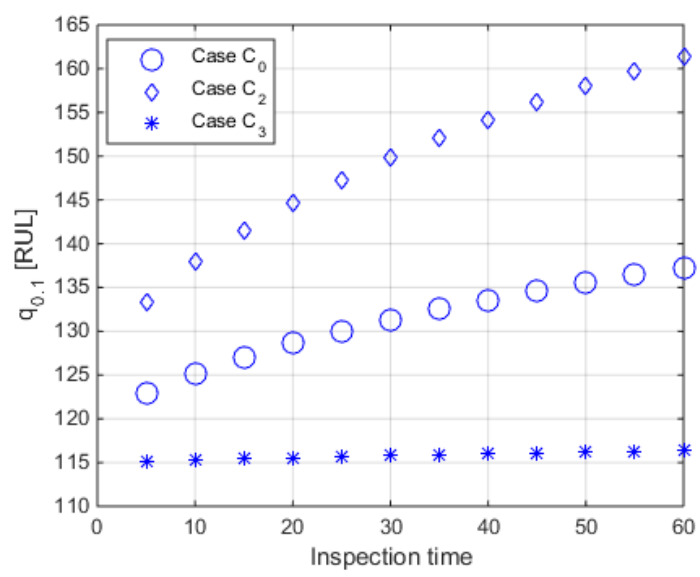

Figure 20: Results for case $C_{0}, C_{2}$ and $C_{3}$ state $1 ; q_{0.1}[R U L]$ and Inspection time in years.

Figures 21 compares the results of case $C_{0}$ to those of cases $C_{2}$ and $C_{3}$, in case the PS is found in state 2. We can note that, initially, the intervals estimated in case $C_{0}$ almost contain the fuzzy estimates of case $C_{2}$ and are very close to those of case $C_{3}$. Then, after $k \tau=25$ years, the estimates of case $C_{3}$ are smaller and differ from the others. This is due to the fact that initially the influence of the expert opinion is more relevant: in fact the estimates of case $C_{3}$, in which the expert is risk-prone, are larger than those of cases $C_{0}$ and $C_{2}$. Figure 22 shows the RUL estimates in cases $C_{0}$ and $C_{3}$, assuming that the component is found in state 3 . In this case, the estimates are closer to each other and sometimes the intervals estimates of case $C_{0}$ contain the possibility distributions of cases $C_{2}$ and $C_{3}$. As for Figure 21 , initially the estimates of case $C_{3}$ are larger than those of cases $C_{2}$ and $C_{0}$, according to the expert opinion. Furthermore, the estimates of cases $C_{2}$ and $C_{3}$ are close to each other since the behavior described by the model parameters, estimated in these two cases, are similar.
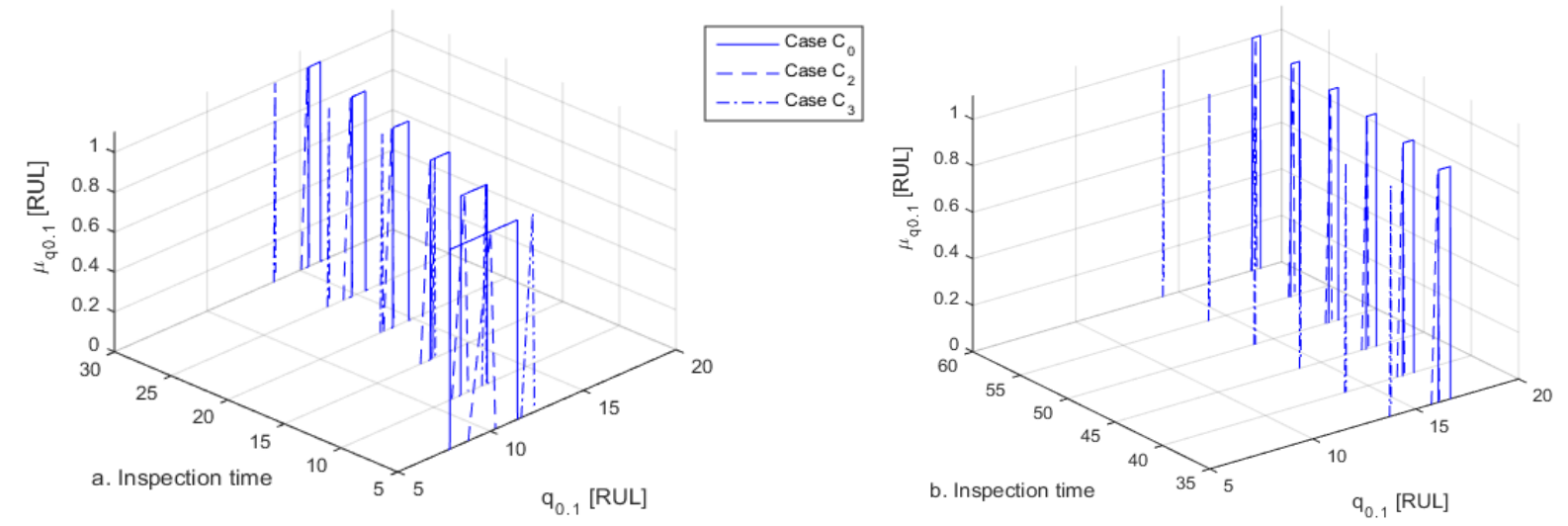
Figure 21: Results for case $C_{0}, C_{2}$ and $C_{3}$, state $2 ; q_{0.1}[R U L]$ and Inspection time in years.
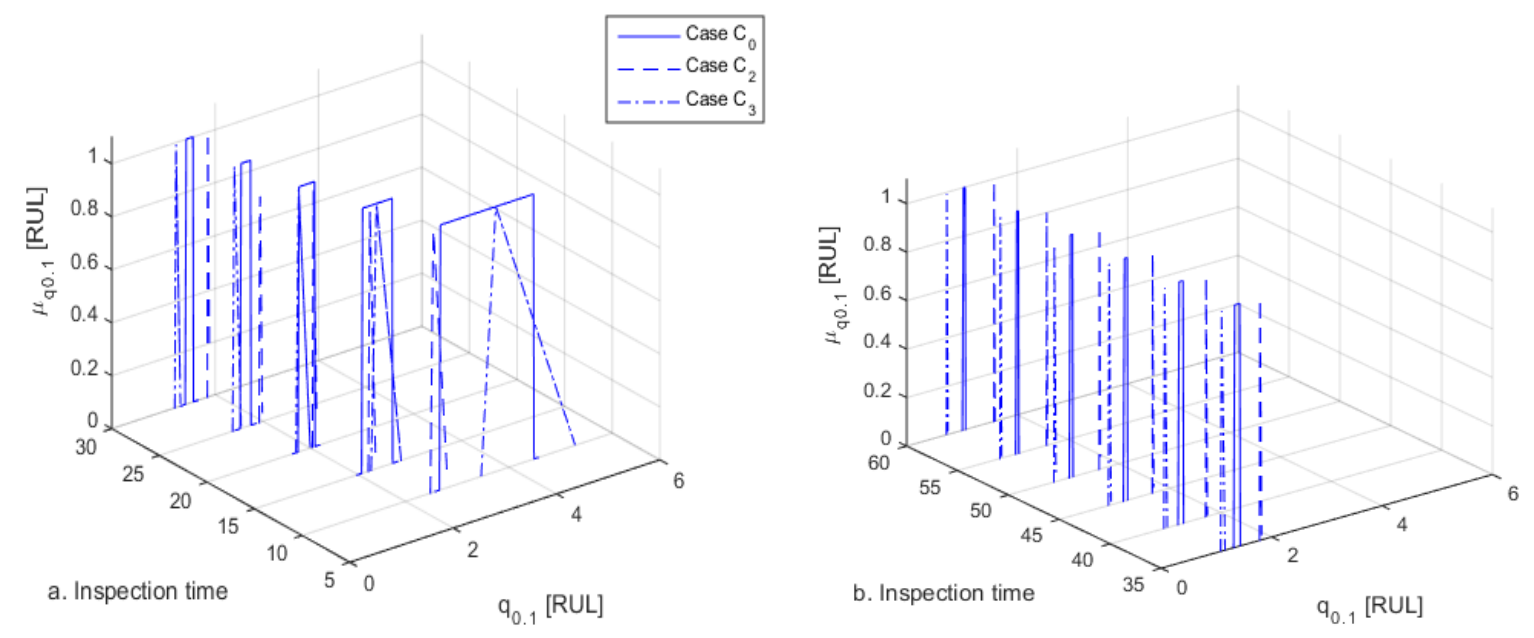

Figure 22: Results for case $C_{0}, C_{2}$ and $C_{3}$, state $3 ; q_{0.1}[R U L]$ and Inspection time in years.

Overall, for all the cases considered, the estimated $E[R U L(k \tau)]$ and $q_{\alpha}[R U L(k \tau)]$ increase proportionally to $k \tau$ when the component is in state 1 or 2 , independently on the case considered, and decrease when the component is in state 3 . This is due to the fact that for $i=1,2 \beta_{i}$ is less than 1 , which corresponds to a decreasing failure rate (Thoman et al., 1969). Consequently, for increasing value of $k \tau, E[R U L(k \tau)]$ and $q_{\alpha}[R U L(k \tau)]$ are increasing over time. On the other hand, for state $i=$ $3, \beta_{3}$ is larger than 1 , which corresponds to an increasing failure rate, and so, for increasing values of $k \tau$, the expected value $E[R U L(k \tau)]$ and the quantile $q_{\alpha}[R U L(k \tau)]$ are decreasing over time.

\section{Conclusions}

In this work, we have developed a method based on the FEM algorithm to estimate the parameters of a MS degradation model. The method allows integrating field data from inspection outcomes with additional information about the state transition times from maintenance operators. Such additional, imprecise information has been represented by possibility distributions. Based on the MS model with estimated parameters, a procedure for predicting the RUL has been developed. The proposed method has been applied to a case study concerning the degradation of pipe welds in the coolant system of a PWR NPP. We have also investigated how results change when the expert knowledge is not employed and only inspection outcomes are considered. The results have shown that the combination of field data with expert knowledge allows reducing the uncertainty in degradation estimation. Finally, the proposed methodology can be easily extended to other industrial reliability problems, where information from expert is available to supplement field data. 


\section{Acknowledgements}

The participation of Enrico Zio to this research is partially supported by the China NSFC under grant number 71231001.

\section{REFERENCES}

Alonso, J.M., Magdalena, L., González-Rodríguez, G., "Looking for a good fuzzy system interpretability index: An experimental approach” International Journal of Approximate Reasoning, 51 (1), pp. 115-134, 2009.

Aven, T., Baraldi, P., Flage, R., Zio, E., "Uncertainty in Risk Assessment: The Representation and Treatment of Uncertainties by Probabilistic and Non-Probabilistic Methods”. Wyley. 2014.

Baraldi, P., Roozbeh R.-F., Zio, E., "Bagged ensemble of Fuzzy C-Means classifiers for nuclear transient identification." Annals of Nuclear Energy 38.5, pp:1161-1171, 2011.

Baudrit, C., Dubois, D., Perrot, N., "Representing parametric models tainted with imprecision. Fuzzy Sets and Systems" Vol. 159, pp. 1913-1928, 2008.

Boutros, T., Liang, M. "Detection and diagnosis of bearing and cutting tool faults using hidden Markov models” Mechanical Systems and Signal Processing, 25 (6), pp. 2102-2124, 2011.

Bowles, J.B., Peláez, C.E., “Application of Fuzzy Logic to Reliability Engineering”, Proceedings of the IEEE, 83 (3), pp. 435-449, 1995.

Cannarile, F., Compare, M., Mattafirri, S., Carlevaro, F., Zio, E., "Comparison of Weibayes and Markov Chain Monte Carlo methods for the reliability analysis of turbine nozzle components with right censored data only", Safety and Reliability of Complex Engineered Systems - Proceedings of the 25th European Safety and Reliability Conference, ESREL 2015, pp. 1937-1944, 2015a.

Cannarile, F., Compare, M., Di Maio, F., Zio, E., "Handling reliability big data: A similarity-based approach for clustering a large fleet of assets", Safety and Reliability of Complex Engineered Systems - Proceedings of the 25th European Safety and Reliability Conference, ESREL 2015, pp. 891-896, 2015b. 
Cannarile, F., Compare, M., Di Maio, F., Zio, E. A Clustering Approach for Mining Reliability Big Data for Asset Management. Proceedings of the Institution of Mechanical Engineers, Part O: Journal of Risk and Reliability, accepted, 2017.

Chapman, O.J.V., Fabbri, L., editors "Discussion document on risk informed in-service inspection of nuclear power plants in Europe" Petten, The Netherlands: European Commission, DG-JRC-Institute for Advanced Materials; 2000.

Chatterjee, K., Modarres, M., “A probabilistic physics of failure approach to prediction of steam generator tube rupture frequency”, ANS PSA 2011 International Topical Meeting on Probabilistic Safety Assessment and Analysis Wilmington, NC, March 13-17, 2011, on CD-ROM, American Nuclear Society, LaGrange Park, 2008.

Compare, M., Baraldi, P., Bani, I., Zio, E., Mc Donnel, D. "Development of a Bayesian multi-state degradation model for up-to-date reliability estimations of working industrial components", Reliability Engineering and System Safety, 166, pp. 25-40, 2017 a.

Compare, M., Baraldi, P., Cannarile, F., Di Maio, F., Zio, E., "Homogeneous finite-time, finite-state, semi-Markov modelling for enhancing Empirical Classification System diagnostics of industrial components", Probabilistic Engineering Mechanics, under review, $2017 \mathrm{~b}$.

Compare, M., Martini, F., Mattafirri, S., Carlevaro, F., Zio, E., "Semi-Markov model for the oxidation degradation mechanism in gas turbine nozzles”, IEEE Transactions and Reliability, 2015, 65(2), pp. 574-581, 2016.

Dempster, A.P., Laird, N.M., Rubin, D.B., "Maximum likelihood from incomplete data via the EM algorithm", Journal of the Royal Statistical Society B, 39, pp. 1-38, 1977.

Denœux, T., "Maximum likelihood estimation from fuzzy data using the EM algorithm" Fuzzy Sets and Systems, 183 (1), pp. 72-9, 2011.

Di Maio, F., Colli, D., Zio, E., Tao, L., Tong, J., “A multi-state physics modelling approach for the reliability assessment of nuclear power plants piping systems" Annals of Nuclear Energy, 80, pp. $151-165,2015$. 
Dubois, D., "Possibility theory and statistical reasoning", Computational Statistics \& Data Analysis, 51, (1), 2006.

Fleming, K.N. "Markov models for evaluating risk-informed in-service inspection strategies for nuclear power plant piping systems", Reliability Engineering and System Safety, 83 (1), pp. 27-45, 2004.

Fleming, K.N., Gosselin S., "Application of Markovian Technique to Modelling influences of inspection on pipe rupture frequencies”, SKI Seminar on Piping Reliability, 1997.

Fleming, K.N., Lydell, B.O.Y., "Database development and uncertainty treatment for estimating pipe failure rates and rupture frequencies", Reliability Engineering and System Safety, 86 (3), pp. 227 246, 2004.

Fleming, K.N., Lydell, B.O.Y., "Pipe rupture frequencies for internal flooding probabilistic Risk Assessment (PRAs)” 101232 (EPRI Licensed Material), Electric Power Research Institute Palo Alto (CA), 2006.

Fleming, K.N., Smit, K. Evaluation of design, leak monitoring, and nde strategies to assure pbmr helium pressure boundary reliability",Proceedings of the 4th International Topical Meeting on High Temperature Reactor Technology, 2, pp. 135-142 HTR 2008.

Ghodrati, B., Farzaneh Ahmadzadeh, Kumar, U., "Mean Residual Life Estimation Considering Operating Environment." International Conference on Quality, Reliability, Infocom Technology and Industrial Technology Management ICQRITTM, 26-28 Nov 2012, Newdelhi, India, 2012.

Giorgio, M., Guida, M., Pulcini, G., “An age- and state-dependent Markov model for degradation processes", IIE Transactions, 43 (9), pp. 621-632, 2011.

Gopika, V., Bidhar, S.K., Kushwaha, H.S., Verma, A.K., Sirividya, A., “A comprehensive framework for evaluation of piping reliability due to erosion corrosion for risk-informed inservice inspection" Reliab. Eng. Syst. Saf. 82, 187-193, 2003. 
Gosselin SR, Fleming KN, "Evaluation of pipe failure potential via degradation mechanism assessment" Fifth International Conference on Nuclear Engineering, May 26-30, Nice, France; 1997.

Howard, R.A. "System Analysis of Semi-Markov Processes", IEEE Transactions on Military Electronics, 8 (2), pp. 114-124, 1964.

Lee, S.-M., Chang, Y.-S., Choi, J.-B., Kim, Y.-J., "Failure probability assessment of wall-thinned nuclear pipes using probabilistic fracture mechanics" Nuclear Engineering and Design, 236 (4), pp. 350-358, 2006.

Limnios, N., Oprisan, G., “Semi-Markov Process and Reliability”, Birkhauser, Boston, 2001.

Lydell, B., Riznic, J., “OPDE-The international pipe failure data exchange project" Nuclear Engineering and Design, 238 (8), pp. 2115-2123, 2008.

Marseguerra, M., Zio, E., Podofillini, L., "A multiobjective genetic algorithm approach to the optimization of the technical specifications of a nuclear safety system" Reliability Engineering and System Safety, 84 (1), pp. 87-99, 2004

Miksch, T.J., Fleming, K.N.,"Piping system failure rates and rupture frequencies for use in risk informed in-service inspection applications” EPRI TR-111880 [ EPRI Licensed Material], 1999.

Moghaddass, R., Zuo, M.J., "An integrated framework for online diagnostic and prognostic health monitoring using a multistate deterioration process" Reliability Engineering an System Safety, 124, pp. 92-104, 2014.

Nánási, T., "Interval censored data analysis with Weibull and exponential distribution” Applied Mechanics and Materials, 693, pp. 74-79, 2014.

Nyman R., Erixon S., Tomic B., Lydell B., "Reliability of Piping System Components" Volume 4: The Pipe Failure Event Database, SKI Report 95:61, 1996.

Rahman, S. "Probabilistic fracture mechanics: J-estimation and finite element methods" Engineering Fracture Mechanics, 68 (1), pp. 107-125, 2001. 
Robert, C.P., Casella, G., "Monte Carlo statistical methods”, New York: Springer, 2004.

Si, X.-S., Wang, W., Hu, C.-H., Zhou, D.-H., "Remaining useful life estimation - A review on the statistical data driven approaches" European Journal of Operational Research, 213 (1), pp. 1-14, 2011.

Simola, K., Pulkkinen, U., Talja, H., Karjalainen-Roikonen, P., Saarenheimo, A., "Comparison of approaches for estimating pipe rupture frequencies for risk-informed in-service inspections", Reliability Engineering and System Safety, 84 (1), pp. 65-74, 2004.

Simonen, F.A., Gosselin, S.R.,'Life prediction and monitoring of nuclear power plant components for service-related degradation", Journal of Pressure Vessel Technology, Transactions of the ASME, 123 (1), pp. 58-64, 2001.

Thoman, D.R., Bain, L.J., Antle, C.E., "Inferences on the Parameters of the Weibull Distribution" Technometrics, 11 (3), pp. 445-460, 1969.

Tipping, G., "Understanding and mitigating ageing in nuclear power plants: Materials and operational aspects of plant life management (PLIM)", Understanding and Mitigating Ageing in Nuclear Power Plants: Materials and Operational Aspects of Plant Life Management (PLIM), pp. 1-914, 2010.

Veeramany, A., Pandey, M.D., "Reliability analysis of nuclear piping system using semi-Markov process model” Annals of Nuclear Energy, 38 (5), pp. 1133-1139, 2011.

Verma, A.K., Srividya, A.K.D., "Risk-Informed Decision Making in Nuclear Power Plants”, Safety and Risk Modeling and its Application, Springer Series in Reliability Engineering, London, 2011.

Vinod, G., Bidhar, S.K., Kushwaha, H.S., Verma, A.K., Srividya, A., “A comprehensive framework for evaluation of piping reliability due to erosion-corrosion for risk-informed inservice inspection", Reliability Engineering and System Safety, 82 (2), pp. 187-193, 2003.

Yuan X., "Stochastic Modeling of Deterioration in Nuclear Power Plant Components" phd thesis, University of Waterloo, 2007. 
Zadeh, L. A., "Fuzzy logic= computing with words." IEEE transactions on fuzzy systems 4. (2), pp. 103-111, 1996.

Zio, E. "An introduction to the basics of reliability and risk analysis" Vol. 13. World scientific, 2007.

Zio, E. "Challenges and opportunities in reliability engineering: the big KID (Knowledge, Information and Data)" IEEE Transactions on Reliability, 65(4), pp. 1769-1782, 2016.

\section{APPENDIX A}

To estimate the reliability function in Figure 10, we have used the following procedure based on Monte Carlo simulation:

\section{Algorithm A:}

While $\operatorname{sim} \leq M$, where $M=100000$ is the number of desired Monte Carlo draws, do:

- Sample sojourn time in state 1: $t_{1}^{\text {sim }} \sim f_{T_{1 \rightarrow 2}}\left(t_{1 \rightarrow 2}, \alpha_{1}, \beta_{1}\right)$

- $\quad \cdots$

- Sample sojourn time in state 3: $t_{3}^{\text {sim }} \sim f_{T_{3 \rightarrow 4}}\left(t_{3 \rightarrow 4}, \alpha_{3}, \beta_{4}\right)$

The quantity $t^{\text {sim }}=\sum_{i=1}^{3} t_{i}^{\text {sim }}$ is a draw from the failure time random variable $T^{\text {failure }}=\sum_{i=1}^{3} T_{i \rightarrow i+1}$. Finally, using draws $t^{\operatorname{sim}}, \operatorname{sim}=1, \ldots, M$, firstly, we have estimated the CDF $F^{\text {failure }}$ of the random variable $T^{\text {failure }}$ with the MATLAB ${ }^{\circledR}$ routine ecdf, then, the reliability function has been estimated as $R^{\text {failure }}=1-F_{\text {failure }}$.

\section{APPENDIX B}

To estimate quantile $q_{\alpha}[R U L(k \tau)]$ we propose the following procedure based on Monte Carlo simulation:

\section{Algorithm B:}

$\mathrm{M}=100000$ is the number of Monte Carlo samples

While $\operatorname{sim} \leq M$ :

- Sample the residual sojourn time in state $i$ provided that the component has already sojourned in this state for $t_{n, i}^{0}$ units of time: $t_{i}^{s i m} \mid t_{n, i}^{0} \sim f_{T_{i \rightarrow i+1}}\left(t_{i \rightarrow i+1} \mid t_{n, i}^{0}, \alpha_{i}, \beta_{i}\right)$,

- For $j=i+1$ to 3

○ Sample sojourn time in state $j: t_{i+1}^{s i m} \sim f_{T_{j \rightarrow j+1}}\left(t_{j \rightarrow j+1}, \alpha_{j}, \beta_{j}\right)$

- End for 


$$
t_{R U L(k \tau)}^{s i m}=t_{i}^{s i m} \mid t_{n, i}^{0}+t_{i+1}^{s i m}+\cdots+t_{3}^{s i m}-t_{n, i}^{0} \text { is a draw from random variable } R U L(k \tau)
$$

End while

Finally, quantile $q_{\alpha}[R U L(k \tau)]$ has been estimated from the collection of samples $t_{R U L(k \tau)}^{\operatorname{sim}}$ with the MATLAB ${ }^{\circledR}$ routine quantile.” 